\title{
Neural entrainment to speech and nonspeech in dyslexia: conceptual replication and extension of previous investigations
}

Mikel Lizarazu1,2, Lou Scotto di Covella1, Virginie van Wassenhove³, Denis Rivière ${ }^{3}$, Raphael Mizzi1,4, Katia Lehongre $^{5}$, Lucie Hertz-Pannier ${ }^{3}$ and Franck Ramus ${ }^{1}$

${ }^{1}$ Laboratoire de Sciences Cognitives et Psycholinguistique, Département d'Etudes Cognitives, Ecole Normale Supérieure, EHESS, CNRS, PSL University, 75005 Paris, France

${ }^{2}$ BCBL, Basque center on Cognition, Brain and Language, Donostia/San Sebastian, Spain

${ }^{3}$ Cognitive Neuroimaging Unit, CEA, INSERM, Université Paris-Sud, Université Paris-Saclay, NeuroSpin center, 91191 Gif/Yvette, France

${ }^{4}$ Laboratoire de Psychologie Cognitive UMR7290, Université Aix-Marseille. 3, Place Victor Hugo, Bat. 9, Case D, 13331 Marseille Cedex 3, France

5 Institut du Cerveau et de la Moelle épinière, Institut National de la Santé et de la Recherche Médicale, Sorbonne Université, 75252 Paris, France

SHORT TITLE: Neural entrainment to speech and nonspeech in dyslexia

KEYWORDS: Dyslexia, Auditory processing, Neural oscillations, Speech, Magnetoencephalography

Corresponding author:

Mikel Lizarazu

Basque Center on Cognition Brain and Language (BCBL)

Mikeletegi Pasealekua, 69

20009 Donostia, Gipuzko, Spain

Email: m.lizarazu@bcbl.eu 


\section{Abstract}

Whether phonological deficits in developmental dyslexia are associated with impaired neural sampling of auditory information is still under debate. Previous findings suggested that dyslexic participants showed atypical neural entrainment to slow and/or fast temporal modulations in speech, which might affect prosodic/syllabic and phonemic processing respectively. However, the large methodological variations across these studies do not allow us to draw clear conclusions on the nature of the entrainment deficit in dyslexia. Using magnetoencephalography, we measured neural entrainment to nonspeech and speech in both groups. We first aimed to conceptually replicate previous studies on auditory entrainment in dyslexia, using the same measurement methods as in previous studies, and also using new measurement methods (cross-correlation analyses) to better characterize the synchronization between stimulus and brain response. We failed to observe any of the significant group differences that had previously been in delta, theta and gamma frequency bands, whether using speech or nonspeech stimuli. However, when analyzing amplitude cross-correlations between noise stimuli and brain responses, we found that control participants showed larger responses than dyslexic participants in the delta range in the right hemisphere and in the gamma range in the left hemisphere. Overall, our results are weakly consistent with the hypothesis that dyslexic individuals show an atypical entrainment to temporal modulations. Our attempt at replicating previously published results highlights the multiple weaknesses of this research area, particularly low statistical power due to small sample size, and the lack of methodological standards inducing considerable heterogeneity of measurement and analysis methods across studies. 


\section{Introduction}

Temporal coding plays a critical role in speech processing and is fundamental to phonological representation, the mental representation of speech sounds. Temporal coding is thought to be accomplished in part by neural entrainment to the temporal modulations of speech at different time scales (Giraud and Poeppel, 2012; Schroeder and Lakatos, 2009; Molinaro and Lizarazu, 2018). Delta (1-3 $\mathrm{Hz}$ ) and theta $(4-7 \mathrm{~Hz})$ oscillations in auditory regions align to prosodic and syllabic rhythms (slow temporal modulations) of speech respectively, while gamma oscillations $(25-60 \mathrm{~Hz})$ track phonemic information (fast temporal modulations) (Gross et al., 2013; Leong and Goswami, 2014; Lizarazu, Lallier and Molinaro, 2019). Furthermore, prior research on the brain bases of temporal sensitivity suggests a right hemisphere preference for processing slow temporal modulations (Poeppel, 2003; Boemio et al., 2005; Abrams et al., 2008; Telkemeyer et al., 2009), and a symmetric pattern for fast modulations (Jamison et al., 2005; Obleser et al., 2008).

Interestingly, previous studies have suggested that a disturbance of auditory entrainment and of the functional hemispheric asymmetries during speech processing may be related to language disorders such as dyslexia (Abrams et al., 2009; Goswami, 2011; Giraud and Poeppel, 2012; Lallier et al., 2017, 2018). It is now well-established that the primary cognitive difficulty found in dyslexia is a difficulty in phonological skills, including phonological awareness, verbal short-term memory and rapid naming (Wagner and Torgesen, 1987). Beyond this consensus, ongoing debates concern whether phonological representations themselves are disrupted (e.g., Noordenbos and Serniclaes 2015) or not (Ramus and Szenkovits, 2008), and whether this phonological deficit originates in defective auditory perceptual processing (see Hämäläinen et al., 2013 for a review) or not (Ramus, 2003; Rosen, 2003). More recently, the study of neural entrainment to temporal modulations has provided a new framework to conceptualize deficits in dyslexia, integrating basic auditory and phonological processing (Goswami, 2011; Giraud and Ramus, 
2013; see Jiménez-Bravo et al., 2017 for a recent review) (view Table 1 for a summary of the main functional studies).

On the one hand, Goswami (2011) has hypothesized that dyslexic readers might show atypical neural entrainment in the delta and theta bands, thereby leading to processing deficits at the prosodic and syllable levels. In the delta band, the dyslexic brain may exhibit weaker entrainment during the processing of speech sounds in right auditory regions, and reduced or absent right-hemisphere lateralization (Molinaro et al., 2016; Power et al., 2013, 2016). Abnormal phase entrainment in the delta range has also been observed during the processing of nonspeech auditory signals in dyslexic participants (Hämäläinen et al., 2012). Hämäläinen and colleagues (2012) measured the phase of the auditory steady-state responses (ASSR) to the presentation of white noise amplitude modulated (AM) at 2, 4, 10 and $20 \mathrm{~Hz}$ in control and dyslexic readers. They found that dyslexic readers exhibited weaker ASSR phase to $2 \mathrm{~Hz}$ AM white noise in right auditory regions. In addition, only control participants showed larger ASSR phase in right than in left auditory regions, indicating more bilateral entrainment in participants with dyslexia. More recently, using functional near-infrared spectroscopy (fNIRS), Cutini and colleagues (2016) reported that dyslexic readers showed atypical $\mathrm{HbO}$ (oxygenated hemoglobin) concentration indices during the processing of $2 \mathrm{~Hz}$ AM white noise in the right supramarginal gyrus - a region suggested to be involved in the processing of speech rhythm and prosody (Geiser et al., 2008). In the theta band, evidence for an abnormal neural entrainment in the auditory regions of dyslexic participants is less conclusive. Increased ASSR phase (Lizarazu et al., 2015) and decrease ASSR power (De Vos et al., 2017) in bilateral auditory cortex has been reported in dyslexic readers compared to controls during the processing of $4 \mathrm{~Hz} \mathrm{AM}$ white noise. No evidence for a disruption in the theta band was found during the processing of speech sounds (Lehongre et al., 2013; Molinaro et al., 2016; Power et al., 2016). 
On the other hand, Giraud and Poeppel (2012) have hypothesized that dyslexic readers might show impaired entrainment in the gamma band, which might disrupt the representation of or the access to phonemic units. Using nonspeech audio signals (AM white noise), McAnally and Stein (1997) showed reduced ASSR power to AMs from 20 to $80 \mathrm{~Hz}$ in dyslexic readers compared to controls. Menell et al. (1999) also measured the ASSR power to 10, 20, 40, 80 and $160 \mathrm{~Hz}$ AMs and showed that the ASSR power was weaker in dyslexic readers compared to controls at all AM rates. However, these studies were unable to examine possible differences in scalp distribution between groups and AM rates, because a single electrode was used to measure ASSR. Lehongre et al. (2011) measured the power of the ASSR in response to white noise across a broad range of amplitude modulations $(10-80 \mathrm{~Hz})$ and showed that the ASSR power at $30 \mathrm{~Hz}$ was weaker in left auditory regions for dyslexic readers compared to controls. Similarly, using whole scalp EEG recording, Poelmans et al. (2012) also reported reduced ASSR power to $20 \mathrm{~Hz}$ AM speechweighted noise in the left hemisphere in dyslexic readers compared to controls. Additionally, Poelmans et al., (2012) found that the inter-hemispheric phase synchronization (IHPS) at $20 \mathrm{~Hz}$ was weaker in dyslexic readers compared to controls (but see De Vos et al., 2017). Poelmans et al. (2012) suggested that reduced phase coherence between distant neural ensembles could compromise the information transfer between left and right auditory regions involved in phonemic sampling. Concerning speech processing, Lehongre and colleagues (2013) reported that dyslexic readers showed reduced gamma oscillatory responses in the left hemisphere during passive viewing of an audiovisual movie.

Overall, published studies have investigated auditory entrainment in dyslexia in multiple frequency bands (delta, theta and beta/gamma), using various stimuli (speech and nonspeech sounds), recording techniques (EEG, MEG, fMRI/EEG, fNIRS), targeting different aspects of auditory entrainment (amplitude and phase responses) and different methods to measure them (power, phase-locking value, interhemispheric phase synchronization, coherence). It is therefore very difficult to have a clear view to what extent the results published in this area are consistent or not, or are simply not comparable. Table 1 
summarizes existing studies, indicating as precisely as possible, the nature of the results reported, as a function of recording technique, number of participants, mean age, language spoken by the participants stimulus type, measurement type and analysis method.

\section{------- Insert Table 1 around here --------}

From Table 1, it appears that methodological variations are the rule, and direct replications the exception. There is therefore a need to replicate previously published results more closely.

The stimuli used to elicit neural entrainment vary considerably across studies. Within the nonspeech stimuli, there are studies using AM white noise at specific rates (McAnally and Stein, 1997; Menell et al., 1999; Hämäläinen et al., 2012; Lizarazu et al., 2015; Cutini et al., 2016; De Vos et al., 2017), AM white noise at rates that increased progressively (Lehongre et al., 2011) or speech-weighted AM noise (Poelmans et al., 2012). Within the speech stimuli, there are studies that used syllables (Power et al., 2013), audiovisual continuous speech (Lehongre et al., 2013), sentences (Molinaro et al., 2016) or noise vocoded sentences (Power et al., 2016).

The measures used to evaluate the neural entrainment also vary importantly across studies. Most of the studies measured power or phase-locking (phase consistency across trials) of the ASSR. Power and phase ASSR measures evaluate the stability of the oscillatory entrainment by rhythmic stimuli, but they are not indicators of the relationship strength between the auditory signal and the neural responses. Coherence is a direct measure of the phase synchronization between the stimulus envelope and the brain oscillations. Cross-correlation analysis can be used to estimate both amplitude and phase speech-brain synchronization (Gross et al., 2013). Furthermore, this method can also estimate the time lag of the maximum entrainment value, which gives a measure of the timing of stimulus envelope processing. Unfortunately, no previous study on dyslexia used cross-correlation measures. 
Most of the studies did not used natural speech as stimuli. As mentioned in Table 1, some studies used AM speech weighted-noise (i.e., Poelmans et al., 2012) or noise vocoded sentences (Power et al., 2016). The temporal modulations of this type of stimuli are close to those of the envelope of the speech signal, but not to those of speech itself. Real speech is only partly modulated, at multiple frequencies simultaneously, and at varying frequencies over time. None of this is reproduced by AM noise, whether speech-weighted or not. Only noise-vocoded speech reproduces such characteristics, progressively becoming more speech-like as the number of channels increases. Furthermore, multiple previous studies suggest that backward speech is processed partly differently from forward speech, even though it has almost the same temporal and spectral properties. Thus, speech has properties that are distinct from any other stimulus, warranting its greater use in dyslexia ASSR studies, despite all the difficulties associated with ecological uncontrolled stimuli. Finally, we observed that most of the studies did not analyze the neural entrainment to both slow (delta and theta) and fast (gamma) temporal modulations. Some studies only tested slow rates (Hämäläinen et al., 2012; Power et al., 2013: Molinaro et al., 2016), whereas other studies only evaluated fast rates (McAnally and Stein, 1997; Menell et al., 1999; Lehongre et al., 2011; Poelmans et al., 2012).

The general goal of the present study is to better understand the neural oscillatory bases underlying the phonological difficulties in dyslexia. First, we aimed to identify the specific frequency band(s) that are affected in dyslexia. Second, we aimed to clarify whether the entrainment difficulties in dyslexia are domain-general or domain-specific i.e. present for speech and nonspeech. We addressed these questions using the measurement method that we think best addressed the working hypotheses under consideration (i.e., cross-correlation of amplitude and of phase responses), and by computing the measures used in previous studies (Table 1) in order to directly compare our results with prior reports. Finally, we tested the link between cortical oscillations and auditory perception in dyslexia. Indeed, the 
putative role of auditory cortical oscillations in auditory processing has until now remained an untested hypothesis.

To answer these questions, we collected behavioral, functional brain activity and structural neuroanatomical data from dyslexic readers and matched controls. We used MEG to record brain activity from control and dyslexic readers while they listened to nonspeech (stationary white noise and white noise $\mathrm{AM}$ at 2, 5 and $30 \mathrm{~Hz}$ ) and speech (forward and backward speech) auditory signals. We evaluated the amplitude and phase synchronization between the envelope of the acoustic signals and the evoked neural oscillations in auditory regions at delta, theta and gamma frequency bands.

We generally hypothesized that auditory neural entrainment would be disrupted in dyslexia, impacting different oscillatory regimes and displaying hemispheric asymmetries. According to Goswami (2011), the disruption was expected in frequency regimes relevant to prosodic (delta) and syllabic (theta) processing. According to Giraud and Poeppel (2012), the disruption was expected mostly in the left hemisphere, in frequency regimes relevant to phonemic processing (gamma). Impairments in neural entrainment were assumed to arise from a primary sensory deficit in auditory rhythm perception, which in turn would affect temporal coding and phonological processing. This working hypothesis predicts that neural entrainment difficulties at a specific frequency band in dyslexia may be present during the processing of both speech and nonspeech stimuli. Finally, we hypothesized that measures of auditory entrainment and their hemispheric lateralization patterns for slow and fast processing may be related to behavioral scores (results from reading, phonological and AM detection tasks). Participants with better behavioral scores were predicted to present stronger specialization of the right and left auditory regions for slow and fast temporal processing, respectively. 


\section{Methods}

The initial analysis plan was pre-registered before analyzing the data. The pre-registration of the analysis of neural entrainment to nonspeech is available under the following link: https://osf.io/wa6mf. The preregistration of the analysis of neural entrainment to speech is available under the following link: https://osf.io/a97n5.

\section{Participants}

Nineteen dyslexic (8 females) and 20 control (12 females) participants matched in age $(t(35)=-0.91$, $p=0.37$, age range: $19-40.7)$ participated in this study. Inclusion criteria required participants (a) to be a native French speaker; (b) to report no neurological/psychiatric disorders; (c) not to be under the influence of psychoactive drugs, (d) to have normal or corrected-to-normal vision and no hearing impairment and (e) to have a non-verbal IQ greater than or equal to 85. Most participants underwent an audiogram screening to check that they could hear tones at 25dB SPL. Unfortunately, this was not done systematically for all participants. For control participants, the crucial criteria were to report no reading/oral language difficulties and to present reading scores above the 10th percentile of their age group's scores in standardized reading tests. For participants with dyslexia, the crucial criteria were a history of reading difficulties and a reading score below the 10th percentile of their age group's scores in standardized reading tests. All participants underwent a diagnostic battery during a preliminary session to ensure that they met inclusion criteria. Experimental tests took place in a separate session. The protocol was approved by the local ethics committee (CPP IDF VII) and each participant signed an informed consent form.

\section{Behavioral data}

\section{Intelligence quotient}


Participants were administered four subtests of the Wechsler Adult Intelligence Scale (WAIS-III FR) battery

(Wechsler, 2008) in order to measure the verbal (vocabulary and similarities subtests) and the non-verbal (picture completion and matrices subtests) intelligence quotient.

\section{Reading skills}

Reading level was assessed via two standardized French tests, "I'Alouette" and "Le Pollueur". L'Alouette test (Lefavrais, 1967) consists in reading aloud a text without meaning. It contains 265 words and includes rare words as well as orthographic and semantic traps. Participants are instructed to read this text as quickly and as accurately as possible. Reading is stopped after 3 minutes. Le Pollueur test (ECLA-16+; GolaAsmussen et al., 2010) contains 296 words and must be read as accurately and quickly as possible. In both tests, we obtained the number of correctly read words per minute (CRWM) by combining total reading time and reading errors. Standardized z-scores were computed based on the mean and standard deviation across all participants.

Furthermore, single word reading skills were assessed through a reading task of regular, non-regular and pseudo-words lists (20 items per list). Number or errors were measured, and z-scores were computed based on the mean and standard deviation across all participants.

\section{Orthographic skills}

Orthographic skills were assessed through dictations (text and single-words) and a computerized orthographic choice task. The single-word dictation consisted of three 10 word-lists of regular, inconsistent and pseudo-words. The text dictation consisted of 78 words and was carried out without time pressure. Errors made on 10 common words and 10 grammatical words were measured. The computerized orthographic choice task consisted in the display of three words, all with the same pronunciation, but only one correctly spelt. The location of the correct word and the two misspelled distractors randomly varied. Participants were asked to select the correct word as fast and accurately as 
possible by pressing one of three arrow keys. A single score was obtained by combining accuracy and response time (1000* proportion of correct answer / response time in ms).

\section{Phonological skills}

Phonological working memory was assessed through a computerized version of the digit-span (WAIS III, Wechsler, 2000) and a pseudo-word repetition computer task. This task consisted of the auditory presentation of one pseudo-word per trial, either of 5 syllables in the first block, of 7 syllables in the second block. Participants were asked to repeat the item out loud. Correct pronunciation was scored 0/1 by the experimenter.

Phonological awareness was assessed through two computer tasks. A first task consisted in the auditory presentation of regular words, to which participants were asked to remove the first sound and to pronounce out loud the new word thus created (i.e., if heard "river", say "iver"). All participants underwent a 4-word training before the 10-word evaluation block. Errors and time were measured. The second task was a spoonerism task. It consisted in the auditory presentation of pairs of regular words. Participants were asked to swap the initial phonemes of the two words, then to pronounce the new (pseudo)words thus created. All participants underwent a 4 trials of training before the 10 -word evaluation block. Errors and time were measured.

Naming fluency was assessed with a rapid automatized naming test. It consisted in naming out loud the items of two series of 50 objects, two of 50 digits, and two of 50 colors. The total time taken to name each entire series was measured.

\section{Musical practice}

Musical practice was evaluated with a set of questions about the musical training (theoretical knowledge and/or instrument practicing) of participants and the number of years of practice was recorded. 


\section{Amplitude Modulation detection task}

Stimuli consisted of amplitude modulated (AM) Gaussian white noise (bandpass filtered $80-8000 \mathrm{~Hz}$ ), generated using Matlab with a sampling frequency of $44.1 \mathrm{kHz}$. In each trial, two $500 \mathrm{~ms}$ stimuli were monaurally presented at $75 \mathrm{~dB}$ SPL: one stationary white noise and one AM white noise (modulation rates were 4,32 or $64 \mathrm{~Hz}$, in each ear, thus yielding 6 conditions). Stimuli were normalized by peak amplitude to ensure equal maximum volume for all stimuli. Peak normalization was based on the highest signal level present in each stimuli. Both signals were presented in pseudo-random order with an inter-stimulus interval of 500 ms. Participants were then asked which sound was modulated, and they had to click on one of two numbered boxes presented on screen. The inter-trial interval was set to one second. In every block, modulation depth was gradually decreased on a per-participant basis using a 1-up-2-down staircase adaptive procedure. Starting from 100\%, modulation was then multiplied (one up) or divided (two down) by 1.58 , corresponding approximately to steps of $4 \mathrm{~dB}$. This value changed to $1.26(2 \mathrm{~dB})$ after the first two reversals. The experiment started with a 5-trial practice for each frequency condition. Participants then underwent 12 experimental blocks, pseudo-randomly varying the side of the stimulated ear and the modulation frequency. Each block lasted until there were 16 reversals or a maximum 150 trials. The mean threshold (in $\mathrm{dB}$ ) was calculated across the values of the last 10 reversals for each condition. Participants were offered to have a short break every four blocks.

Participants were tested individually in a double-walled IAC sound booth, and responded with either a computer mouse or a trackpad. Stimuli were presented monaurally through Sennheiser HD 600 headphones at $70 \mathrm{~dB}$.

\section{Structural MRI}

\section{Data acquisition}


All subjects underwent structural MRI scanning in a single session, using the Magnetom Terra 7 Tesla system (Siemens AG, Erlangen, Germany), located at the Neurospin center. A high-resolution T1-weighted scan was acquired with a 3D ultrafast gradient echo (MPRAGE) pulse sequence using a 32-channel head coil and with the following acquisition parameters: $\mathrm{FOV}=256 ; 160$ contiguous slices, $\mathrm{TR}=14 \mathrm{~ms}, \mathrm{TE}=3,06$ $\mathrm{ms}, \mathrm{BW}=250 \mathrm{~Hz} /$ pixel, acceleration factor $=3$, flip angle variable $=5-9 \mathrm{deg}$, voxel resolution $=1 \times 1 \times 1$ $\mathrm{mm}$.

\section{Functional data (MEG recordings)}

\section{Stimuli and Procedure}

The nonspeech stimuli were generated using Matlab with a sampling frequency of $44.1 \mathrm{kHz}$. The nonspeech stimuli consisted of amplitude modulated white noise at three different frequencies: $2 \mathrm{~Hz}, 5$ $\mathrm{Hz}$ and $30 \mathrm{~Hz}$ with $100 \%$ depth. In addition, a control condition included stationary white noise. The duration of the stimuli varied as a function of the AM frequency: $3 \mathrm{~s}$ for the $2 \mathrm{~Hz}$ ( 6 cycles), $1.6 \mathrm{~s}$ for the 5 $\mathrm{Hz}$ (8 cycles) and $0.6 \mathrm{~s}$ for the $30 \mathrm{~Hz}$ (18 cycles) AM rate to guarantee a minimum number of cycles while minimizing time. The duration for the stationary white noise was $0.6 \mathrm{~s}$. There were 100 trials for each of the AM white noise conditions.

The speech stimuli consisted of forward and backward speech. For the forward speech condition, fiftyfour sentences were uttered by a French female speaker and digitized at $16 \mathrm{kHz}$. The length of the sentences was between 15 and 21 syllables and the mean duration of the sentences was $2.89 \mathrm{~s}$ (Min=2.17s; Max=3.92s: SD=0.36s). As a control condition, the same sentences were played backwards (backward speech condition).

During the MEG recording, stimuli were presented pseudo-randomly in 5 separate blocks with an interstimulus interval (ISI) varying randomly between $2 \mathrm{~s}$ and $3 \mathrm{~s}$. Auditory stimuli were delivered to both ears 
using Matlab via Etymotic earphones. The sound level was fixed at $75 \mathrm{~dB}$ SPL. Participants were asked to look at a fixation cross, and avoid head movements and blinks during the presentation of the stimuli.

\section{Data acquisition and pre-processing}

MEG data were acquired in a magnetically shielded room using the whole-scalp MEG system (ElektaNeuromag, Helsinki, Finland) installed at Neurospin (CEA Saclay). The system is equipped with a helmetshaped array of 306 sensors, arranged in triplets of two orthogonal planar gradiometers and a magnetometer. The position of the head with respect to the sensor array was estimated at the beginning of each block using five Head Position Indicator (HPI) coils. A 3D digitizer (Fastrak Polhemus, Colchester, VA, USA) was used to define the location of each HPI coil and approximately 100 "headpoints" along the scalp, relative to the anatomical fiducials (the nasion and left and right preauricular points). Digitization of the fiducials plus $\sim 100$ additional points evenly distributed over the scalp of the participant were used for coregistration. Data were recorded at a sampling rate of $1 \mathrm{KHz}$ and filtered on-line with a bandwidth of $0.01-330 \mathrm{~Hz}$. Eye movements were monitored with two pairs of electrodes in a bipolar montage placed on the external corner of each eye (horizontal electrooculography (EOG)) and above and below the right eye (vertical EOG). Cardiac rhythms were recorded using three electrodes (ECG) - one on the right side of the subjects' abdomen, one on the left lower rib and one below the left clavicle.

The continuous MEG data from each block were processed off-line using MNE-Python (Gramfort et al., 2013). The Signal-Space-Separation (SSS) method (Taulu, Simola and Kajola, 2005) was applied in order to reduce environmental and biological noise. The MEG data was aligned across blocks to match the head position at the start of the first run. Data from different blocks was concatenated into a single file and low-pass filtered at $80 \mathrm{~Hz}$ for each participant. Heart beat and EOG artifacts were detected using Independent Component Analysis (ICA) and linearly subtracted from concatenated file. The ICA decomposition was performed using the FastICA algorithm (Hyvärinen and Oja, 2000). Subsequent 
analyses were performed using Matlab R2010 (MathWorks). Raw data were segmented into epochs from $1.5 \mathrm{~s}$ before the stimulus presentation (pre-stimulus interval) to the duration of each stimulus (poststimulus interval). Epochs with large MEG peak-to-peak amplitude values (exceeding 3e-12 $\mathrm{T}$ in magnetometer or $3000 \mathrm{e}-13 \mathrm{~T} / \mathrm{m}$ in gradiometers) were considered as artifact contaminated and rejected from the subsequent analyses.

\section{Source activity estimation}

Using the MNE suite, the digitized points from the Polhemus were co-registered to the skin surface. We used an anatomically realistic three-shell model to calculate the forward solution. Individual T1-weigthed MRI images were segmented into scalp, skull, and brain compartments using the segmentation algorithms implemented in Freesurfer (Martinos Center of Biomedical Imaging, MQ). The noise covariance matrix was estimated from the empty room data acquired right before bringing the subject in the MEG room. We used the noise covariance matrix to whiten the forward matrix and the data (Lin et al., 2006; Lütkenhöner, 1998). The sources of the MEG signals were estimated in the individual's brain using L2 minimum-norm estimates (MNE) (Hämäläinen and Ilmoniemi, 1994). Functional brain measures were obtained in the individual's brain and transformed to the standard Montreal Neurological Institute (MNI) brain using the spatial-normalization algorithm implemented in Statistical Parametric Mapping (SPM8, Wellcome Department of Cognitive Neurology, London, UK). We followed this procedure for each participant, condition and time-point. Group-level statistics were computed in the MNI space.

\section{Localization of auditory areas}

We analyzed the MEG neural response to stationary white-noise to localize auditory regions in all participants. The average source activity in the post-stimulus interval $(0.1-0.3 \mathrm{~s})$ was compared to the average source activity in a time window of the same length within the pre-stimulus interval $\left(\begin{array}{ll}-0.2-0 & s\end{array}\right)$ using a permutation cluster t-test corrected for multiple comparisons (Nichols and Holmes, 2002) (as done 
in Lizarazu et al., 2015). Cortical sources showing significantly larger responses $(p<0.01)$ in the poststimulus period compared to the pre-stimulus interval were grouped to create regions of interest (ROIs). Based on previous studies, we expected to find bilateral auditory regions as ROIs.

\section{Neural entrainment measures}

Neural entrainment to different auditory stimuli was evaluated using different methods (signal-to-noise ratio (SNR), phase locking value (PLV), inter-hemispheric phase synchronization (IHPS), coherence and phase/amplitude cross-correlation analysis). Furthermore, for each measurement of entrainment the lateralization index (LI) was computed to evaluate the functional hemispheric asymmetries during the processing of different stimuli.

\section{Signal-to-noise ratio}

The power of the auditory-steady state response (ASSR) to 2, 5 and $30 \mathrm{~Hz}$ AMs was estimated based on signal-to-noise ratio (SNR) of cortical evoked fields (Poelmans et al., 2012). The power analysis evaluated the strength of the oscillatory response to the AMs at different rates. Artifact-free epochs were averaged and the power spectrum was estimated in the post-stimulus interval using fast Fourier transform. To estimate the noise-power $\left(P_{N}\right)$ we obtained the mean power of the Fourier components in an approximately $2 \mathrm{~Hz}$ wide frequency band around the modulation frequency (i.e. approximately $1 \mathrm{~Hz}$ on each side). The SNR was calculated between the response power $\left(P_{S}\right)$ and the $P_{N}$ (John and Picton, 2000):

$$
S N R=10 \times \log _{10}\left(\frac{P_{S}}{P_{N}}\right)
$$

The SNR was calculated for each source in the left and the right auditory regions. Finally, mean of SNR values was obtained in the left and the right auditory regions. 
In order to test for group differences, we computed an ANOVA on the mean SNR values, with Condition (2, 5 and $30 \mathrm{~Hz})$ and Hemisphere (left and right) as the within-subject factors and Group (dyslexic and control participants) as between-subject factor.

\section{Phase Locking Value}

The phase consistency of the auditory steady-state responses (ASSR) to 2, 5 and $30 \mathrm{~Hz}$ AMs was estimated using Phase Locking Value (PLV) (Hämäläinen et al., 2012; Lizarazu et al., 2015). PLV is also known as phase locking factor (PLF), mean phase coherence (MPC) or inter-trial phase coherence (ITPC). The PLV gives an estimate of how consistently the phase of the oscillatory activity in the MEG response follows the AM at each rate $(2,5$ and $30 \mathrm{~Hz})$ across the recording. The PLV was computed in the post-stimulus intervals using a sliding window of duration corresponding to two modulation cycles with $50 \%$ overlap. The PLV was calculated as follows:

$$
P L V=\frac{1}{N}\left|\sum_{n=1}^{N} e^{i \theta_{n}}\right|
$$

where $n$ is the phase of the source activity for the $n^{t h}$ widow and $N$ is the total number of windows. If the phase was perfectly aligned across trials the value was 1 , and if the phase was perfectly random across trials the value is 0 . The PLV was calculated for each source in the left and the right auditory regions. Finally, mean of PLVs was obtained in the left and the right auditory regions.

In order to test for group differences, we computed an ANOVA on the mean PLVs, with Condition (2, 5 and $30 \mathrm{~Hz}$ ) and Hemisphere (left and right) as the within-subject factors and Group (dyslexic and control participants) as between-subject factor.

\section{Inter-hemispheric phase synchronization}

The inter-hemispheric phase synchronization (IHPS) was calculated to estimate the stimulus-driven synchronization between left and right auditory regions (Poelmans et al., 2012). We computed the inter- 
hemispheric phase synchronization in the post-stimulus intervals using a sliding window of duration corresponding to two modulation cycles with $50 \%$ overlap. It was calculated substituting $\theta_{n}=\theta_{R_{n}}-$ $\theta_{L_{n}}$ in formula 2. In this case, $\theta_{R_{n}}$ and $\theta_{L_{n}}$ were the mean phase of the source activity for the $n^{\text {th }}$ window in the left and the right auditory regions.

In order to test for group differences, we computed an ANOVA on the mean IHPS values, with Condition $(2,5$ and $30 \mathrm{~Hz})$ as the within-subject factors and Group (dyslexic and control participants) as betweensubject factor.

\section{Coherence}

We used coherence to evaluate the phase synchronization between neural oscillations and the envelope signals (Molinaro et al., 2016; Molinaro and Lizarazu, 2018; Lizarazu, Lallier and Molinaro, 2019). The envelope of the speech was estimated by using a filter bank that models the passage of the signal through the cochlea (Kösem et al., 2016; Ghitza 2011). For each experimental condition, coherence between the artifact free epochs and the audio signals was calculated in the $0.5-15 \mathrm{~Hz}$ frequency band with $0.5 \mathrm{~Hz}$ frequency resolution. Coherence was estimated for each source in the left and right auditory regions. Then, the mean coherence within the left and the right auditory regions was obtained.

We first identified the specific frequency bands that showed significant coherence values during the forward speech processing in all participants. The statistical significance of coherence values was determined using a surrogate data analysis. Surrogate coherence values were created by computing coherence between the auditory oscillations elicited by the forward speech condition and randomly selected parts of the reversed speech. This procedure was repeated 500 times and the coherence value across frequencies was selected to define a surrogate data distribution. This provides an estimate of the coherence values that can be expected by chance. Frequencies for which the non-randomized coherence values exceeded the 95th percentile of this surrogate distribution were defined as frequencies of interest. 
Contiguous significant frequencies were grouped in frequency bands of interest. Based on previous studies, we expected to find significant coherence values in the delta and theta frequency bands. Finally, mean of the coherence values was obtained in each frequency band in the left and the right auditory regions for each participant and condition. In order to test for group differences, we computed an ANOVA on the mean coherence values, with Condition (forward and backward), Frequency band (delta and theta bands) and Hemisphere (left and right) as the within-subject factors and Group (dyslexic and control participants) as between-subject factor.

\section{Cross-correlation analysis}

We used cross-correlation analysis to estimate both amplitude and phase synchronization between the neural oscillations and the speech envelope (Gross et al., 2013).

The envelope of the audio signals was estimated by using a filter bank that models the passage of the signal through the cochlea (Kösem et al., 2016; Ghitza 2011). Brain signals were bandpass filtered in the 1

- $40 \mathrm{~Hz}$ frequency band with $1 \mathrm{~Hz}$ frequency resolution (fourth order Butterworth filter, forward and reverse, center frequency $\pm 1 \mathrm{~Hz}$, or $\pm 5 \mathrm{~Hz}$ for frequencies above $40 \mathrm{~Hz}$ ). Amplitude and phase dynamics of the MEG trials were computed using Hilbert transform for each bandpass signal. The cross-correlation of either the cosine of the phase (cos(phase)) or the amplitude with the corresponding audio envelope was computed over time with various lags in steps of $5 \mathrm{~ms}$ up to a maximum of $150 \mathrm{~ms}$ for each trial. For each lag, the correlation values were averaged across trials in the same condition and across sources in the ROIs. Finally, we measured the maximum correlation $\left(\rho_{\text {max }}\right)$ across lags and the corresponding lag value $(\tau)$. This was done in order to adjust the analysis to each participant's individual apparent latency between auditory stimulation and cortical response.

For the nonspeech stimuli, mean of the phase/amplitude $\rho_{\max }$ and $\tau$ values were obtained around the target $A M$ frequency ( $A M$ frequency $\pm 1 \mathrm{~Hz}$ ) in the left and the right auditory regions. In order to test for 
group differences in neural entrainment to nonspeech, separate ANOVAs were computed on the mean phase/amplitude $\rho_{\max }$ and $\tau$ values, with Condition $(2,5$ and $30 \mathrm{~Hz})$ and Hemisphere (left vs. right) as the within-subject factors and Group (dyslexic vs. control participants) as between-subject factor.

For the forward speech stimuli, we first identified the specific frequency bands that showed significant phase/amplitude $\rho_{\max }$ values during the forward speech processing in all participants. The statistical significance of phase/amplitude $\rho_{\max }$ values was determined using a surrogate data analysis (Gross et al., 2013). Surrogate phase/amplitude $\rho_{\max }$ values were created by computing cross-correlation between the auditory oscillations elicited by the forward speech condition and randomly selected parts of the reversed speech. This procedure was repeated 500 times and the maximum phase/amplitude $\rho_{\max }$ value across frequencies was selected to define a surrogate data distribution. This provides an estimate of the phase/amplitude $\rho_{\max }$ values that can be expected by chance. Frequencies for which the non-randomized phase/amplitude $\rho_{\max }$ values exceeded the 95 percentile of this surrogate distribution were defined as frequencies of interest. Contiguous significant frequencies were grouped in frequency bands of interest. Based on previous studies (Gross et al., 2013), we expected to find significant phase $\rho_{\max }$ correlation values within the delta and theta frequency bands, and significant amplitude $\rho_{\max }$ values within the gamma band. Finally, mean of the phase/amplitude $\rho_{\max }$ and $\tau$ values were obtained in each frequency band in the left and the right auditory regions for each participant and condition.

In order to test for group differences, separate ANOVAs were computed on the mean of the phase/amplitude $\rho_{\max }$ and $\tau$ values, with Condition (forward and backward), Frequency (delta and theta) and Hemisphere (left and right) as the within-subject factors and Group (dyslexic and control participants) as between-subject factor.

\section{$\underline{\text { Lateralization Index }}$}


When a main effect or an interaction with hemisphere emerged, the lateralization index (LI) values were analyzed. The LI was calculated for each dependent variable (amplitude/phase $\rho_{\text {max }}$ and $\tau$ values):

$$
L I=\frac{A_{R}-A_{L}}{A_{R}+A_{L}}
$$

where $A_{R}$ and $A_{L}$ refers to the mean of each measured variable within the ROI of the right and left hemisphere respectively. This formula renders positive $\mathrm{LI}$ values for right-dominance and negative values for left-dominance. The LI values were tested against zero with a one-sample t-test to determine a left or right significant lateralization for a specific group, condition and frequency band. Group differences on the $\mathrm{LI}$ values were assessed using a two-sample t-test.

\section{Correlation analysis}

We obtained three indices from the behavioral data: the literacy index, the phonological index and the RAN index. For the literacy index, the z-scores of the measures obtained in the reading (l'alouette, le pollueur, word reading) and the orthographic (word and text dictation and tri ortho) tasks were averaged. For the phonological index, the z-scores of the measures obtained in the phonological working memory and the phonological awareness tasks were averaged. For the RAN index, the z-scores of the RAN objects, digits and colors were averaged. Pearson's correlations between the behavioral data and the brain measures showing significant group differences were computed. Correlation between the scores of AM detection task and the brain measures showing significant group differences were also computed. 


\section{Results}

\section{Behavioral results}

The characteristics of control and dyslexic participants are presented in Supplementary Table 1.

The verbal and non-verbal IQ scores on the WAIS test were superior to 85 in all participants, suggesting normal intelligence in all our participants. The verbal IQ was significantly lower in dyslexic participants compared to controls, whereas no significant differences emerged in regard to the non-verbal IQ.

Dyslexic readers showed low reading performance compared to controls. The "Alouette" and the "Le Pollueur" tests showed that the correctly read words per minute (CRWM) scores (both the raw and the ztransformed values) were significantly lower in controls compared to dyslexic participants. For the word list reading task, dyslexic readers showed more difficulties reading regular, irregular and pseudo-words.

Orthographic processing skills were impaired in dyslexic readers. The dyslexic group made more errors than controls in word (regular, irregular and pseudo-words) and text (spelling and grammar) dictation. Dyslexic readers were also slower and less accurate than controls in the trio-ortho computer task.

Dyslexic participants showed weaker phonological processing skills: indeed, they obtained significantly poorer performance than controls in the tasks that assessed phonological working memory (digit-span and pseudo-word repetition), phonological awareness (Phonemic deletion and Spoonerism) and Rapid Automatized Naming (RAN) of objects, digits and colors.

Musical practice was highly variable, but comparable between groups with $6.47 \pm 6.74$ and $3 \pm 4.71$ years of practice for control and dyslexic participants, respectively.

In the behavioral AM task, we measured an AM detection threshold for each participant in the three frequency conditions. We computed an ANOVA on detection thresholds with Frequency $(4 \mathrm{~Hz}, 32 \mathrm{~Hz}$ and 
$64 \mathrm{~Hz}$ ) as a within-participant factor and Group (Control or Dyslexic) as a between-participant factor. The main Group effect was not significant $\left(F(1,31)=2.22, p=0.15, \eta_{p}^{2}=0.07\right)$, failing to show different thresholds between control $(11.12 \pm 4.03 \mathrm{~dB})$ and dyslexic $(12.54 \pm 5.56 \mathrm{~dB})$ participants. However, the main Frequency effect appeared significant $\left(F(2,62)=43.9, p<0.01, \eta_{p}^{2}=0.58\right)$ with $4 \mathrm{~Hz}$ thresholds $(16.1 \pm 6.08$ $\mathrm{dB}$ ) higher than in the $64 \mathrm{~Hz}$ frequency condition $(10.73 \pm 2.62 \mathrm{~dB})$, themselves higher than in the $32 \mathrm{~Hz}$ condition $\left(8.81 \pm 1.71 \mathrm{~dB}\right.$ ). Post-hoc tests revealed that these differences were significant (all $p_{\text {bonferroni(bonf.) }}$ $<0.01)$. Interestingly, the interaction between the two main factors showed a trend toward significance $\left(F(2,62)=2.5, p=0.09, \eta_{p}^{2}=0.08\right)$. The $4 \mathrm{~Hz}$ frequency condition showed the largest difference between groups (14.26 $\pm 5.11 \mathrm{~dB}$ and $17.67 \pm 6.6 \mathrm{~dB}$ for the control and dyslexic groups respectively, $\mathrm{d}=0.58$ ) while both $32 \mathrm{~Hz}$ and $64 \mathrm{~Hz}$ showed comparable thresholds ( $8.68 \pm 1.56$ and $8.8 \pm 1.93$ for the $32 \mathrm{~Hz}$ condition, $10.29 \pm 2.38$ and $11.14 \pm 2.14$ for the $64 \mathrm{~Hz}$ condition). However, no difference between groups appeared in post-hoc tests (all $p_{\text {bonf }}>0.1$ ).

\section{Brain functional results}

\section{Localization of auditory areas}

First, we analyzed the auditory responses evoked by the presentation of the stationary white noise to localize auditory cortices in all participants (Supplementary Figure 1). Power values were significantly higher in post-stimulus $(0.1-0.3 \mathrm{~s})$ compared to the pre-stimulus $(-0.2-0)$ interval in bilateral auditory region, including mainly Brodmann areas 41 and 42 (primary auditory regions). These are the regions of interest (ROIs) for subsequent analyses.

\section{Conceptual replication and extension of previous neural entrainment results}

Supplementary Table 2 reports the means and standard deviations of the signal-to-noise ratio (SNR), phase locking value (PLV), inter-hemispheric phase synchronization (IHPS) and coherence values in the left and right auditory cortices for control and dyslexic participants. 


\section{Nonspeech stimuli}

The power of the ASSR evoked by the white noise AM at 2, 5 and $30 \mathrm{~Hz}$ were estimated by measuring the SNR of cortical evoked fields (Supplementary Figure 2 and 3A). Supplementary Figure 2 shows the power spectrum density of the ASSR at different AM rates in left and right auditory cortices for all participants. We observed that the SNR strongly peaked around the AM frequency for different conditions. Results of the ANOVA (Group $x$ Condition $x$ Hemisphere) for the SNR values showed a Condition by Hemisphere interaction $\left(F(1,37)=3.04, p=0.05, \eta_{p}^{2}=0.07\right)$. The Lateralization Index (LI) analysis reveals that the SNR values were significantly right lateralized at $2 \mathrm{~Hz}(\mathrm{LI}=0.11, p=0.05)$ and were bilateral at $5 \mathrm{~Hz}(\mathrm{LI}=-0.001$, $p=0.49)$ and $30 \mathrm{~Hz}(\mathrm{LI}=-0.02, p=0.39) \mathrm{AM}$.

The phase locking values of the ASSR in response to the white noise AM at 2,5 and $30 \mathrm{~Hz}$ was estimated using PLV (Supplementary Figure 3B) The ANOVA (Group $x$ Condition $x$ Hemisphere) of the PLVs showed a main effect of Condition $\left(F(2,74)=101.1, p<0.01, \eta_{p}^{2}=0.73\right)$. Post-hoc tests showed that PLVs were significantly higher at $2 \mathrm{~Hz}$ compared to $5 \mathrm{~Hz}\left(t(76)=4.19, p_{\text {bonf }}<0.01, d=0.67\right)$, at $2 \mathrm{~Hz}$ compared to $30 \mathrm{~Hz}$ $\left(t(76)=12.92, p_{\text {bonf }}<0.01, d=2.07\right)$ and at $5 \mathrm{~Hz}$ compared to $30 \mathrm{~Hz}\left(t(76)=10.7, p_{\text {bonf }}<0.01, d=1.71\right)$. We also found a main effect of Hemisphere $\left(F(1,37)=16.24, p<0.01, \eta_{p}^{2}=0.3\right)$. The PLVs were significantly higher in the right compared to left auditory cortex $\left(t(115)=3.97, p_{\text {bonf }}<0.01, d=0.64\right)$. The $\mathrm{LI}$ analysis reveals that the phase PLVs were significantly right lateralized at $2 \mathrm{~Hz}(\mathrm{LI}=0.07, p<0.01), 5 \mathrm{~Hz}(\mathrm{LI}=0.05, p=0.02)$ and 30 $\mathrm{Hz}(\mathrm{LI}=0.07, p<0.01) \mathrm{AM}$.

Auditory inter-hemispheric synchronization during the processing of the white noise AM at 2, 5 and $30 \mathrm{~Hz}$ was estimated using inter-hemispheric phase synchronization (IHPS). The ANOVA (Group x Condition) of the IHPS values showed a main effect of Frequency $\left(F(2,74)=3.79, p=0.03, \eta_{p}^{2}=0.09\right)$. Post-hoc tests showed that PLVs were significantly higher at $30 \mathrm{~Hz}$ compared to $5 \mathrm{~Hz}\left(t(76)=3.05, p_{\text {bonf }}=0.01, d=0.49\right)$.

\section{Speech stimuli}


We used coherence to estimate the phase synchronization between the cortical oscillations in auditory regions and the speech envelope (Supplementary Figure 4 and 5). In Supplementary Figure 4 we observed that coherence values were significantly higher for the forward speech condition compared to the surrogate data in the delta $(0.5-2.5 \mathrm{~Hz})$ and the theta $(3.5-6 \mathrm{~Hz})$ frequency bands in all participants. The ANOVA (Group $x$ Frequency band $x$ Hemisphere) of the mean coherence values showed a main effect of Frequency band $\left(F(1,37)=28.03, p<0.01, \eta_{p}^{2}=0.41\right)$. Post-hoc tests showed that coherence values were higher in the delta band compared to the theta band $\left(t(76)=5.05, p_{\text {bonf }}<0.01, d=0.81\right)$. We also observed a main effect of Condition $\left(F(1,37)=6.77, p=0.01, \eta_{p}^{2}=0.15\right)$ and a Frequency band by Condition interaction $\left(F(1,37)=6.77, p=0.01, \eta_{p}^{2}=0.15\right)$. The coherence values were higher for the forward speech compared to the backward speech in the theta band $\left(t(154)=5.02, p_{\text {bonf }}<0.01, d=0.8\right)$, but not in the delta band $\left(t(154)=0.05, p_{\text {bonf }}=0.96, d<0.01\right)$. The results also revealed a significant interaction between the Condition and the Hemisphere factors $\left(F(1,37)=5.61, p=0.02, \eta_{p}^{2}=0.13\right)$. The $\mathrm{LI}$ analysis showed that coherence values were right lateralized for the forward speech condition $(L I=0.06, p=0.02)$ and were bilateral for the backward speech condition $(\mathrm{LI}=-0.02, p=0.24)$.

\section{Conceptual replication of previous studies}

Furthermore, we tested the various predictions made by previous studies using the corresponding entrainment measurement. Results of the statistics (independent sample t-tests) are included in Table 2. Some of the previous studies could not be replicated (Power et al., 2013, 2016; Lehongre et al., 2013; Cutini et al., 2016; Molinaro et al., 2016) because methodological differences with our study were too great.

Insert Table 2 around here

Overall, we failed to replicate previous results suggesting a group difference in the delta, theta and gamma ranges, whether measured using SNR, PLV, IHPS or coherence. However, we did replicate previously 
reported null results concerning the delta and theta ranges. Our single result that comes close to the replication of a positive result is the trend towards a lower SNR in dyslexics in the theta range $(t(76)=1.41, p=0.07, d=0.31)$, previously reported by De Vos et al. (2017).

\section{Cross-correlation analysis}

\section{Nonspeech stimuli}

We found that phase and amplitude $\rho_{\max }$ values strongly peaked around the AM frequency in the left and the right auditory cortex in all participants (Supplementary Figure 6). Phase and amplitude maximum correlation $\left(\rho_{\max }\right)$ and the corresponding lag value $(\tau)$ were obtained at the modulation rate for each condition in the left and right auditory regions in control and dyslexic participants (Figure 1, 2and Supplementary Table 3 and 4).

For the phase $\rho_{\max }$ values (Figure 1A), results of the ANOVA (Group $x$ Condition $x$ Hemisphere) showed a main effect of Condition $\left(F(2,74)=11455.58, p<0.01, \eta_{p}^{2}=0.99\right)$. Post-hoc tests showed that phase $\rho_{\max }$ values were significantly higher at $30 \mathrm{~Hz}$ compared to $2 \mathrm{~Hz} \operatorname{AM}\left(t(76)=182.55, p_{\text {bonf }}<0.01, d=29.23\right)$, at 30 $\mathrm{Hz}$ compared to $5 \mathrm{~Hz} \mathrm{AM}\left(t(76)=102.59, p_{\text {bonf }}<0.01, d=16.43\right)$, and at $5 \mathrm{~Hz}$ AM compared to $2 \mathrm{~Hz} \mathrm{AM}$ $\left(t(76)=36.93, p_{\text {bonf }}<0.01, d=5.91\right)$. We also found a main effect of Hemisphere $(F(1,37)=7.33, p=0.01$, $\left.\eta_{p}^{2}=0.17\right)$. Overall, the phase $\rho_{\max }$ values were significantly higher in the right compared to the left auditory cortex $\left(t(115)=2.75, p_{\text {bonf }}<0.01, d=0.44\right)$. We also found a Condition by Hemisphere interaction $\left(F(1,37)=3.81, p=0.03, \eta_{p}^{2}=0.09\right)$. The Lateralization Index (LI) analysis, revealed that the phase $\rho_{\max }$ values were right lateralized at $2 \mathrm{~Hz} \mathrm{AM} \mathrm{(LI=0.013,p<0.01)}$ and bilateral at $5 \mathrm{~Hz}(\mathrm{LI}=0.003, p=0.16)$ and $30 \mathrm{~Hz} \mathrm{AM}$ (LII=-0.001, $p=0.24)$.

For the phase $\tau$ values (Figure 1B), results of the ANOVA (Group $x$ Condition $x$ Hemisphere) showed a main effect of Condition $\left(F(2,74)=8.13, p<0.01, \eta_{p}^{2}=0.17\right)$. Post-hoc tests showed that phase $\tau$ values were 
significantly higher at $30 \mathrm{~Hz}$ compared to $2 \mathrm{~Hz} \mathrm{AM}\left(t(76)=3.05, p_{\text {bonf }}=0.01, d=0.49\right)$, at $30 \mathrm{~Hz}$ compared to $5 \mathrm{~Hz}$ AM $\left(t(76)=4.28, p_{\text {bonf }}<0.01, d=0.68\right)$.

\section{------- Insert Figure 1 around here}

For the amplitude $\rho_{\max }$ values (Figure $2 \mathrm{~A}$ ), results of the ANOVA (Group $\times$ Condition $x$ Hemisphere) showed a main effect of Condition $\left(F(2,74)=346.38, p<0.01, \eta_{p}^{2}=0.9\right)$. Post-hoc tests showed that amplitude $\rho_{\max }$ values were significantly higher at $2 \mathrm{~Hz}$ compared to $5 \mathrm{~Hz} \operatorname{AM}\left(t(76)=10.95, p_{\text {bonf }}<0.01, d=1.75\right)$, at $2 \mathrm{~Hz}$ compared to $30 \mathrm{~Hz} \mathrm{AM}\left(t(76)=22.28, p_{\text {bonf }}<0.01, d=3.57\right)$, and at $5 \mathrm{~Hz}$ compared to $30 \mathrm{~Hz} \mathrm{AM}(t(76)=36.44$, $\left.p_{\text {bonf }}<0.01, d=5.83\right)$. We also found a main effect of Hemisphere $\left(F(1,37)=9.47, p<0.01, \eta_{p}^{2}=0.19\right)$. Overall, the amplitude $\rho_{\max }$ values were significantly higher in the left compared to the right auditory cortex $\left(t(115)=3.01, p_{\text {bonf }}<0.01, d=0.48\right)$. Interestingly, we found a Condition by Hemisphere interaction $\left(F(2,74)=6.71, p<0.01, \eta_{p}^{2}=0.13\right)$ and a Condition by Hemisphere by Group interaction $(F(2,74)=6.52$, $\left.p<0.01, \eta_{p}^{2}=0.13\right)$. Post hoc tests showed that amplitude $\rho_{\max }$ values were significantly higher for controls compared to dyslexic participants at $30 \mathrm{~Hz}$ in the left hemisphere $\left(t(37)=3.31, p_{\text {bonf }}<0.01, d=1.06\right)$. Amplitude $\rho_{\max }$ values were also marginally higher for controls compared to dyslexic participants at $2 \mathrm{~Hz}$ in the right hemisphere $\left(t(37)=1.59, p_{\text {bonf }}=0.06, d=0.51\right)$. In control participants, the LI analysis revealed that amplitude $\rho_{\max }$ values were left lateralized at $30 \mathrm{~Hz}$ AM $(\mathrm{LI}=-0.01, p<0.01)$, and bilateral at $2 \mathrm{~Hz}(\mathrm{LI}=-$ $0.006, p=0.27)$ and $5 \mathrm{~Hz}$ AM (LI=-0.003, $p=0.27)$. In dyslexic participants, the LI analysis revealed that the


and $30 \mathrm{~Hz} A M(\mathrm{LI}=-0.002, p=0.27)$. The $\mathrm{LI}$ values were significantly different between groups at $2 \mathrm{~Hz}$ $(p=0.01)$ and marginally so at $30 \mathrm{~Hz}(p=0.06)$.

For the amplitude $\tau$ values (Figure 2B), results of the ANOVA (Group $\times$ Condition $x$ Hemisphere) showed a main effect of Condition $\left(F(2,74)=174.15, p<0.01, \eta_{p}^{2}=0.81\right)$. Post-hoc tests showed that amplitude $\tau$ values 
were significantly higher at $30 \mathrm{~Hz}$ compared to $2 \mathrm{~Hz} \mathrm{AM}\left(t(76)=18.01, p_{\text {bonf }}<0.01, d=2.88\right)$ and at $30 \mathrm{~Hz}$ compared to $5 \mathrm{~Hz}$ AM $\left(t(76)=14.44, p_{\text {bonf }}<0.01, d=2.31\right)$.

\section{------- Insert Figure 2 around here}

\section{Speech stimuli}

We evaluated the spectral profile of the phase and amplitude $\rho_{\max }$ values for forward speech in the left and right auditory regions in all participants (Figure 3 and 4, and Supplementary Figure 7).

Phase $\rho_{\max }$ values were significantly higher for forward speech compared to surrogate data in delta ( $1-3$ $\mathrm{Hz}$ ) and theta $\left(4-8 \mathrm{~Hz}\right.$ ) frequency bands (Supplementary Figure $7 \mathrm{~A}$ ). For the phase $\rho_{\max }$ values (Figure 3 and Supplementary Table 3), results of the ANOVA (Group x Condition x Hemisphere) showed a main effect of Frequency band $\left(F(1,37)=13649.52, p<0.01, \eta_{p}^{2}=0.99\right)$ and Hemisphere $(F(1,37)=6.41, p=0.02$, $\left.\eta_{p}^{2}=0.15\right)$. Post-hoc tests showed that phase $\rho_{\max }$ values were significantly higher in the delta band compared to the theta band $(t(76)=116.7, p<0.01, d=18.69)$. Furthermore, phase $\rho_{\max }$ values were significantly higher in the right than in the left auditory cortex $(t(155)=2.757, p=0.01, d=0.41)$. We also observed a main effect of Condition $\left(F(1,37)=5.17, p=0.03, \eta_{p}^{2}=0.12\right)$ and a Frequency band by Condition interaction $\left(F(1,37)=8.21, p<0.01, \eta_{p}^{2}=0.18\right)$. The phase $\rho_{\max }$ values were higher for forward speech than for backward speech in the delta band $\left(t(154)=3.79 p_{\text {bonf }}<0.01, d=0.6\right)$, but not in the theta band $(t(154)=1$, $p_{\text {bonf }}=0.32, d=0.16$ ). For phase $\tau$ values, results of the ANOVA (Group $\mathrm{x}$ Speech condition $\mathrm{x}$ Hemisphere $\mathrm{x}$ Frequency band) showed no main effects or interactions (all $F s<3.65, p s>0.06, \eta_{p}^{2}=<0.09$ ).

\section{------- Insert Figure 3 around here}

Amplitude $\rho_{\max }$ values were significantly higher for forward speech than for surrogate data in the delta $(0.5-3 \mathrm{~Hz})$, theta $(4-7 \mathrm{~Hz})$, alpha $(8-11 \mathrm{~Hz})$, beta $(18-22 \mathrm{~Hz})$ and gamma $(33-36 \mathrm{~Hz})$ frequency bands (Supplementary Figure 7B). 
For the amplitude $\rho_{\max }$ values (Figure 4), results of the ANOVA (Group $x$ Speech condition $x$ Hemisphere $x$ Frequency band) showed a main effect of Frequency $\left(F(4,148)=31.5, p<0.01, \eta_{p}^{2}=0.46\right)$ band. Post-hoc test showed that amplitude $\rho_{\max }$ values were significantly higher at delta compared to beta $(t(76)=7.41$, $p<0.01, d=1.19)$ and gamma $(t(76)=9.55, p<0.01, d=1.53)$ bands, at theta compared to beta $(t(76)=7.47$, $p<0.01, d=1.2)$ and gamma $(t(76)=9, p<0.01, d=1.44)$ bands, at alpha compared to beta $(t(76)=4.57$, $p<0.01, d=0.73)$ and gamma $(t(76)=5.51, p<0.01, d=0.88)$ bands, and at beta compared to gamma band $(t(76)=3.9, p<0.01, d=0.62)$. No other main effects or interactions were found (all $F s<2.88, p s>0.05$, $\left.\eta_{p}^{2}<0.06\right)$. For comparison with the results obtained on the amplitude $\rho_{\max }$ values for the nonspeech stimuli, amplitude $\rho_{\max }$ values for the speech stimuli were compared between groups at delta in the right hemisphere and at gamma in the left hemisphere separately for each speech condition. We did not observe significant group differences in any of the comparisons (all $t s(37)<1.03, p s>0.31, d s<0.33$ ).

For the phase $\tau$ values, results of the ANOVA (Group $x$ Speech condition $x$ Hemisphere $x$ Frequency band) showed no main effects or interactions (all $F s<2.16, p s>0.08, \eta_{p}^{2}<0.05$ ).

\section{------- Insert Figure 4 around here}

\section{Correlation between behavioral and functional results}

Table 4 reports the correlation analysis between the behavioral scores and the brain measures showing significant group differences, i.e., amplitude cross correlations for $2 \mathrm{~Hz}$ AM noise in right auditory cortex, and for $30 \mathrm{~Hz}$ AM noise in left auditory cortex. Significant correlations are plotted in Figure 8.

------- Insert Table 3 around here

------- Insert Figure 5 around here 


\section{Discussion}

In the present study, we have used MEG to measure neural entrainment to speech and nonspeech sounds in dyslexic and normal-reading adult individuals. We have attempted to conceptually replicate previously published results on auditory entrainment in dyslexia, using the same measurement methods as in previous studies, and using new measurement methods in order to better capture the synchronization between stimulus and brain response.

\section{Results of general interest}

Before turning to group differences between dyslexic and control participants, let us discuss a number of results that we have obtained that are of general interest for our understanding of the neural bases of speech perception. Some of these results are consistent with previous investigations, and some are entirely new.

We find that neural responses entrained by the amplitude modulations of auditory stimuli are localized mostly around auditory cortex, in particular in Brodmann areas 41 and 42 bilaterally, including primary and secondary cortex. Of course, this attribution of responses to specific neuroanatomical areas is subject to the limits of spatial resolution in MEG. When the stimulus is amplitude-modulated at one specific frequency, neural responses closely follow at the same frequency. When the stimulus is natural speech, including modulations in many frequency bands, neural responses reflect the predominant modulations present in speech, notably in the delta, theta and gamma ranges. In those fundamental matters, the different measures investigated, whether power, phase-locking, coherence, or cross-correlation, are largely concordant. Generally speaking, measures of power and amplitude showed stronger signal at lower frequencies (delta and theta) and weaker signal at higher frequencies (gamma), so much so that gamma responses to speech stimuli did not emerge from noise, except for amplitude cross-correlations. 
Different measures of phase synchronization gave contrasting results: phase-locking values were stronger in the delta and theta than in the gamma ranges, but phase cross-correlations for noise and interhemispheric phase locking values were stronger in gamma than in theta and delta ranges. Phase cross-correlations between speech and brain were also much stronger in the delta than in the theta range.

\section{Latency}

We estimated the apparent latency between stimulus and neural response, by determining the time lag between stimulus and response at which cross-correlations were maximal, both in the phase and in the amplitude domains. We found time lags between 60 and $80 \mathrm{~ms}$, which is consistent with previous findings (Thwaites et al., 2015; Bourguignon et al., 2018). Phase time lags were slightly higher in the gamma than in delta and theta ranges for noise, but overall values were quite similar across frequencies. Amplitude time lags showed a clearer trend in the same direction, being about 5 ms longer in the gamma than in delta-theta ranges. Latency did not differ between the two hemispheres.

\section{Hemispheric lateralization:}

While the responses we have observed are generally bilateral, a number of them have shown to be stronger in one hemisphere than in the other. In particular:

- $\quad$ SNR values were significantly right lateralized at $2 \mathrm{~Hz}$ and were bilateral at 5 and $30 \mathrm{~Hz} \mathrm{AM}$.

- Phase-locking was stronger in the right than in the left hemisphere, in all frequency bands.

- Phase cross-correlations between noise and brain were right-lateralized at $2 \mathrm{~Hz}$ and bilateral at 5 and $30 \mathrm{~Hz}$ AM. Similarly, phase cross-correlations between speech and brain (in delta and theta ranges) were significantly higher in the right than in the left auditory cortex.

- Amplitude cross-correlations between noise and brain were left-lateralized at $30 \mathrm{~Hz}$ and bilateral at 2 and $5 \mathrm{~Hz}$ AM. 
Finally, inter-hemispheric synchronization for AM noise seems to be stronger in the gamma than in the delta and theta ranges.

\section{Forward vs. backward speech:}

- The coherence of neural responses to speech stimuli was higher for forward than for backward speech, in the theta but not in the delta band. This is consistent with the idea that backward speech preserves the rhythm of speech (delta range) but not its smaller scale regularities (at the syllabic level, theta range), where the inversion of syllables' envelope makes them distinctly nonspeech-like and may disrupt speech-specific processing (Mehler et al., 1988; Ramus et al., 2000). However, phase cross correlations between speech and brain were higher for forward than for backward speech in the delta band, but not in the theta band.

- Whereas responses to forward speech in the delta and theta ranges were right-lateralized, responses to backward speech were bilateral. This is consistent with the idea that hemispheric lateralization implements some processing mechanisms that are specific to speech sounds.

\section{Interest of cross-correlation measures}

The various brain measures used in previous studies (SNR, PLV and IHPS) all provide information on the quality and consistency of brain responses. However, they do not address the crucial question whether neural responses faithfully represent the incoming stimuli, and whether they do so sufficiently rapidly. Cross-correlation captures precisely the extent to which neural responses reflect the stimuli, both in phase and in amplitude.

Furthermore, we also measured the latency between the stimulus and the response that yields the maximal cross-correlation. This is interesting to the extent that latency might differ in certain pathologies, such as in dyslexia. 
Finally, when standard amplitude measures were insensitive to frequencies beyond the theta range in brain responses to speech, amplitude cross-correlations proved to be sensitive to brain responses in alpha, beta, and gamma frequency bands.

Nevertheless, we do not mean to claim that cross-correlation is the ultimate measure of neural synchronisation with auditory signals. In the future, more sophisticated techniques such as temporal response function (Crosse et al. 2016) might prove even more fruitful.

\section{Conceptual replication of previous studies on dyslexia}

Our attempt at replicating previously published group differences has largely failed. We failed to observe any of the significant group differences that have previously been reported in the delta, theta and gamma ranges, whether using speech or AM white noise stimuli, and whether using signal-to-noise ratio, phaselocking value, coherence, or interhemispheric phase synchronization measures. However, we replicated previously reported null differences, mostly in the delta and theta ranges using all the different measures.

It may be argued that, with 39 participants, we had limited power to detect previously reported effects. This is entirely true. Indeed, in simple group comparisons, we had $80 \%$ power to detect a $d=0.92$ effect size, which is large and probably implausible. However, while our statistical power is obviously insufficient in hindsight, it was not so initially. Indeed, half of previous studies did not have larger samples than ours, and some reported quite large effect sizes (Table 1). We therefore made a fair attempt at replicating the largest reported effects.

Another possible reason for our failure to replicate previous results lies in many methodological differences.

- In the present study, MEG has been considered as the technique of choice for the investigation of neural entrainment to auditory signals. One of the main advantages of MEG and EEG over fMRI 
and fNIRS techniques is their excellent temporal resolution, of the order of milliseconds (Hämäläinen et al., 1993). This high temporal resolution enables the investigation of fast variations in cortical activity (i.e., gamma band). In addition, fMRI or fNIRS measure indirect correlates of neural activity, such as the neurometabolic or neurovascular coupling, whereas EEG and MEG techniques directly measure electromagnetic neural activity. Compared to EEG, MEG has a higher spatial resolution and is particularly well suited for source localization procedures (i.e. identification of auditory regions) (Destoky et al., 2019).

- In the current study, we restricted the analysis to the auditory areas using a localization procedure. We found that the auditory areas mainly covered BA41 and BA42 (primary auditory regions). However, previous studies were not so precise in the way they defined auditory regions. There are EEG studies that used a single electrode (McAnally and Stein, 1997; Menell et al., 1999) or a small number of electrodes (8 electrodes) (Poelmans et al., 2012) to measure neural entrainment to audio signals in control and dyslexic participants. De Vos and colleagues (2017) selected a set of electrodes located in left and right temporo-parietal hemispheric regions to evaluate neural entrainment. These studies were probably measuring neural activity from auditory regions, but also neural activity from other regions that may add noise to the entrainment measurement. There are also studies that looked for whole-brain group differences on the neural entrainment values (Cutini et al., 2016; Molinaro et al., 2016). When computing whole brain statistical analysis, we increase the odd of finding some spurious false positive just by chance.

As can be seen, the present study is not an identical replication of any previous study. Indeed, there are not two studies in this literature that identically replicate each other. Thus, our study is at best a conceptual replication, and it is a closer conceptual replication of some studies (Lehongre et al., 2011; Hämäläinen et al., 2012) than of others (Lizarazu et al., 2015; De Vos et al., 2017). 
To these specific reasons why replication may have failed, it is worth adding more general reasons, that cut across all experimental science: the fact that most published studies are based on relatively small samples, that are adequately powered only to detect the largest effect sizes, which are generally implausible. Thus, when such studies happen to find a significant effect, there is a high risk that it might be a false positive result, hence the difficulty to replicate it (Button et al. 2013). Furthermore, as we have highlighted, there are many ways to measure auditory entrainment, involving sophisticated analysis techniques that require a large number of decisions to be made and parameters to be set. This gives investigators many degrees of freedom to explore different analysis variants until a publishable result is found, further increasing the risk of obtaining a false positive result. Because the measurement of auditory entrainment is a relatively young area, such degrees of freedom are not reduced by established standards. Similar considerations have been made in many different areas, including for instance in the study of neuroanatomical differences in dyslexia (Ramus et al. 2018; Jednorog et al. 2015).

In terms of sample size, the present study does not fare better than previous ones. However, because we preregistered it, because we systematically investigated previously used measures according to preregistration, and because we systematically reported all our results rather than selectively reporting the only positive ones, it acts as a revelator of this situation.

It is also important to note that in the present study the hearing (or auditory sensitivity) of the participants was not systematically checked. Inter-individual variation of 20-30 dB in hearing thresholds is very common and might introduce differences in auditory steady state amplitudes for equal stimulus presentation levels, thus increasing statistical noise and reducing the detection of significant differences.

\section{Extension of previous studies on dyslexia}

While previous studies have reported results using certain stimuli, certain frequency ranges and certain response measures, they did not carry out (or at least report) systematic analyses of all the possible 
combinations of stimuli, frequency range and measure (see Table 1). Thus, in a second step, we extended previous studies by analyzing more systematically auditory entrainment in our data, measured using SNR, PLV, IHPS for AM white noise (at 2, 5 and $30 \mathrm{~Hz}$ ), and coherence for sentences (forward and backward). However, none of these analyses showed any difference between dyslexic and control groups.

\section{Cross-correlation analyses}

Finally, we went beyond previous studies by performing analyses of cross-correlations between stimulus and brain response in participants with dyslexia. In order to avoid the previously mentioned pitfalls associated with multiple analysis variants increasing the risk of false positive results, we preregistered this analysis.

Overall, most of our results were similar between control and dyslexic participants. The two groups did not differ in terms of phase cross-correlation between stimulus and response. They did not differ either in terms of the lag of maximal cross-correlation ( $\tau$ values). However, when analyzing amplitude crosscorrelations between noise stimuli and brain responses, we found that dyslexic and control groups differed in their hemispheric lateralization for gamma and delta frequency ranges: controls showed larger responses than dyslexics in the gamma range $(30 \mathrm{~Hz})$ in the left hemisphere, and in the delta range $(2 \mathrm{~Hz})$ in the right hemisphere. While controls showed left lateralized responses at $30 \mathrm{~Hz}$ and bilateral responses at $2 \mathrm{~Hz}$, dyslexic participants showed on the contrary bilateral responses at $30 \mathrm{~Hz}$ and left lateralized responses at $2 \mathrm{~Hz}$.

In the similar analysis of cross-correlations between speech and brain responses, we found no significant group difference across all frequency bands. In left-hemisphere responses in the gamma range, and in right-hemisphere responses in the delta range, we observed similar trends toward group differences as for the noise stimuli, but they were not significant. 
Although this cross-correlation analysis differs from those of previous studies, it remains nevertheless interesting that the group differences observed are consistent with some previous studies: weaker responses in the gamma range in the left hemisphere in dyslexics have previously been reported by McAnally and Stein, 1997, Menell et al., 1999, Lehongre et al., 2011, and Poelmans et al., 2012; weaker responses in the delta range in the right hemisphere in dyslexics has previously been reported by Hämäläinen et al., 2012 and Molinaro et al., 2016.

Thus, we are in the strange position of failing to replicate some results of previous studies when using exactly the same measures, but finding results that seem consistent with them when using different measures. This can only reinforce the feeling that replication is being hindered by power limitations, both in the original studies and in ours, thus giving chance a large role in determining which group differences are significant or not.

Our finding of group differences in neural responses to AM noise, but not in neural responses to speech (despite similar trends), may be interpreted in a similar manner. Stimuli that are generated in such a way as to be $100 \%$ amplitude-modulated at one single frequency can only elicit stronger and more reliable neural responses than speech, which is only partly modulated, at multiple frequencies that vary in time. Thus studies measuring neural responses to AM noise must be more sensitive to group differences than those measuring neural responses to speech. If one takes into account publication bias, this may be one reason for the vast predominance of published studies reporting group differences between dyslexics and controls that use noise compared to those that use speech (as can be seen in Table 1).

\section{Brain-behavior correlations}

Finally, we also carried out correlation analyses between the two amplitude cross-correlation measures that showed group differences ( $30 \mathrm{~Hz}$ in the left and $2 \mathrm{~Hz}$ in the right hemisphere) with the available 
behavioural measures. Some nominally significant correlations were found, but they would not withstand a correction for multiple tests, and do not seem reliable enough to warrant discussion here.

\section{Conclusion}

Overall, our results are weakly consistent with the hypothesis that dyslexic individuals show a slight disruption in the entrainment of their auditory cortex to amplitude-modulated sounds, specifically to 30 $\mathrm{Hz}$ modulations in the left hemisphere and to $2-\mathrm{Hz}$ modulations in the right hemisphere. Thus, they are consistent with theories invoking disruptions of phoneme and of prosodic processing in dyslexia (Giraud \& Poeppel, 2012; Giraud \& Ramus, 2013; Goswami, 2011). However, our attempt at replicating previously published results highlights the multiple weaknesses of this research area, particularly low statistical power due to small sample sizes, and the lack of methodological standards inducing considerable heterogeneity of measurement and analysis methods across studies. Future research on auditory entrainment in dyslexia will need to rely on much larger samples, and to copy more closely the methods used by previous studies, including the cross-correlation analyses that we have used in the present study,

which we think may provide new insights into the neural bases of speech processing and its differences in individuals with dyslexia. 


\section{References}

Abrams, D. A., Nicol, T., Zecker, S., \& Kraus, N. (2008). Right-hemisphere auditory cortex is dominant for coding syllable patterns in speech. Journal of Neuroscience, 28(15), 3958-3965.

Abrams, D. A., Nicol, T., Zecker, S., \& Kraus, N. (2009). Abnormal cortical processing of the syllable rate of speech in poor readers. Journal of Neuroscience, 29(24), 7686-7693.

Boemio, A., Fromm, S., Braun, A., \& Poeppel, D. (2005). Hierarchical and asymmetric temporal sensitivity in human auditory cortices. Nature Neuroscience, 8(3), 389.

Bourguignon, M., Baart, M., Kapnoula, E. C., \& Molinaro, N. (2018). Hearing through lip-reading: the brain synthesizes features of absent speech. bioRxiv, 395483.

Button, K. S., Ioannidis, J. P., Mokrysz, C., Nosek, B. A., Flint, J., Robinson, E. S., \& Munafò, M. R. (2013). Power failure: why small sample size undermines the reliability of neuroscience. Nature Reviews Neuroscience, 14(5), 365.

Crosse, M. J., Di Liberto, G. M., Bednar, A., \& Lalor, E. C. (2016). The multivariate temporal response function (mTRF) toolbox: a MATLAB toolbox for relating neural signals to continuous stimuli. Frontiers in human neuroscience, 10, 604.

Cutini, S., Szűcs, D., Mead, N., Huss, M., \& Goswami, U. (2016). Atypical right hemisphere response to slow temporal modulations in children with developmental dyslexia. Neurolmage, 143, 40-49.

Destoky, F., Philippe, M., Bertels, J., Verhasselt, M., Coquelet, N., Vander Ghinst, M., ... \& Bourguignon, M. (2019). Comparing the potential of MEG and EEG to uncover brain tracking of speech temporal envelope. Neuroimage, 184, 201-213. 
De Vos, A., Vanvooren, S., Vanderauwera, J., Ghesquiere, P., \& Wouters, J. (2017). A longitudinal study investigating neural processing of speech envelope modulation rates in children with (a family risk for) dyslexia. Cortex, 93, 206-219.

Geiser, E., Zaehle, T., Jancke, L., \& Meyer, M. (2008). The neural correlate of speech rhythm as evidenced by metrical speech processing. Journal of Cognitive Neuroscience, 20(3), 541-552.

Ghitza, O. (2011). Linking speech perception and neurophysiology: speech decoding guided by cascaded oscillators locked to the input rhythm. Frontiers in Psychology, 2, 130.

Giraud, A. L., \& Poeppel, D. (2012). Cortical oscillations and speech processing: emerging computational principles and operations. Nature Neuroscience, 15(4), 511.

Giraud, A. L., \& Ramus, F. (2013). Neurogenetics and auditory processing in developmental dyslexia. Current Opinion in Neurobiology, 23(1), 37-42.

Glasberg, B. R., \& Moore, B. C. (1990). Derivation of auditory filter shapes from notched-noise data. Hearing research, 47(1-2), 103-138.

Gola-Asmussen, C., Lequette, C., Pouget, G., Rouyet, C., \& Zorman, M. (2010). Evaluation des Compétences de Lecture chez l'Adulte de plus de 16 ans [Reading Skills Assessment for Adults over age 16].

Goswami, U. (2011). A temporal sampling framework for developmental dyslexia. Trends in Cognitive Sciences, 15(1), 3-10.

Gramfort, A., Luessi, M., Larson, E., Engemann, D. A., Strohmeier, D., Brodbeck, C., ... \& Hämäläinen, M. (2013). MEG and EEG data analysis with MNE-Python. Frontiers in Neuroscience, 7, 267. 
Gross, J., Hoogenboom, N., Thut, G., Schyns, P., Panzeri, S., Belin, P., \& Garrod, S. (2013). Speech rhythms and multiplexed oscillatory sensory coding in the human brain. PLoS Biology, 11(12), e1001752.

Hämäläinen, M., Hari, R., Ilmoniemi, R. J., Knuutila, J., \& Lounasmaa, O. V. (1993). Magnetoencephalography-theory, instrumentation, and applications to noninvasive studies of the working human brain. Reviews of Modern Physics, 65(2), 413.

Hämäläinen, M. S., \& Ilmoniemi, R. J. (1994). Interpreting magnetic fields of the brain: minimum norm estimates. Medical \& Biological Engineering \& Computing, 32(1), 35-42.

Hämäläinen, J. A., Rupp, A., Soltész, F., Szücs, D., \& Goswami, U. (2012). Reduced phase locking to slow amplitude modulation in adults with dyslexia: an MEG study. Neuroimage, 59(3), 2952-2961.

Hämäläinen, J. A., Salminen, H. K., \& Leppänen, P. H. (2013). Basic auditory processing deficits in dyslexia: systematic review of the behavioral and event-related potential/field evidence. Journal of Learning Disabilities, 46(5), 413-427.

Hyvärinen, A., \& Oja, E. (2000). Independent component analysis: algorithms and applications. Neural Networks, 13(4-5), 411-430.

Jamison, H. L., Watkins, K. E., Bishop, D. V., \& Matthews, P. M. (2005). Hemispheric specialization for processing auditory nonspeech stimuli. Cerebral Cortex, 16(9), 1266-1275.

Jednoróg, K., Marchewka, A., Altarelli, I., Monzalvo, K., van Ermingen-Marbach, M., Grande, M., Grabowska, A., Heim, S., \& Ramus, F. (2015). How reliable are grey matter disruptions in specific reading disability across multiple countries and languages? Insights from a large-scale voxel-based morphometry study. Human Brain Mapping, 36, 17411754. 
Jimenez-Bravo, M., Marrero, V., \& Benitez-Burraco, A. (2017). An oscillopathic approach to developmental dyslexia: From genes to speech processing. Behavioural Brain research, 329, 84-95.

John, M. S., \& Picton, T. W. (2000). Human auditory steady-state responses to amplitude-modulated tones: phase and latency measurements. Hearing research, 141(1-2), 57-79.

Kösem, A., Basirat, A., Azizi, L., \& van Wassenhove, V. (2016). High-frequency neural activity predicts word parsing in ambiguous speech streams. Journal of Neurophysiology, 116(6), 2497-2512.

Lallier, M., Molinaro, N., Lizarazu, M., Bourguignon, M., \& Carreiras, M. (2017). Amodal atypical neural oscillatory activity in dyslexia: A cross-linguistic perspective. Clinical Psychological Science, 5(2), 379-401.

Lallier, M., Lizarazu, M., Molinaro, N., Bourguignon, M., Ríos-López, P., \& Carreiras, M. (2018). From auditory rhythm processing to grapheme-to-phoneme conversion: How neural oscillations can shed light on developmental dyslexia. In Reading and Dyslexia (pp. 147-163). Springer, Cham.

Lefavrais, P. (1967). Test de l'Alouette.

Lehongre, K., Ramus, F., Villiermet, N., Schwartz, D., \& Giraud, A. L. (2011). Altered low-gamma sampling in auditory cortex accounts for the three main facets of dyslexia. Neuron, 72(6), 1080-1090.

Lehongre, K., Morillon, B., Giraud, A. L., \& Ramus, F. (2013). Impaired auditory sampling in dyslexia: further evidence from combined fMRI and EEG. Frontiers in Human Neuroscience, 7, 454.

Leong, V., \& Goswami, U. (2014). Assessment of rhythmic entrainment at multiple timescales in dyslexia: evidence for disruption to syllable timing. Hearing research, 308, 141-161.

Lin, F. H., Witzel, T., Ahlfors, S. P., Stufflebeam, S. M., Belliveau, J. W., \& Hämäläinen, M. S. (2006). Assessing and improving the spatial accuracy in MEG source localization by depth-weighted minimumnorm estimates. Neuroimage, 31(1), 160-171. 
Lizarazu, M., Lallier, M., Molinaro, N., Bourguignon, M., Paz-Alonso, P. M., Lerma-Usabiaga, G., \& Carreiras, M. (2015). Developmental evaluation of atypical auditory sampling in dyslexia: Functional and structural evidence. Human Brain Mapping, 36(12), 4986-5002.

Lizarazu, M., Lallier, M., \& Molinaro, N. (2019). Phase- amplitude coupling between theta and gamma oscillations adapts to speech rate. Annals of the New York Academy of Sciences.

Lütkenhöner, B. (1998). Dipole source localization by means of maximum likelihood estimation. I. Theory and simulations. Electroencephalography and Clinical Neurophysiology, 106(4), 314-321.

McAnally, K. I., \& Stein, J. F. (1997). Scalp potentials evoked by amplitude-modulated tones in dyslexia. Journal of Speech, Language, and Hearing Research, 40(4), 939-945.

Menell, P., McAnally, K. I., \& Stein, J. F. (1999). Psychophysical sensitivity and physiological response to amplitude modulation in adult dyslexic listeners. Journal of Speech, Language, and Hearing research, 42(4), 797-803.

Mehler, J., Jusczyk, P., Lambertz, G., Halsted, N., Bertoncini, J., \& Amiel-Tison, C. (1988). A precursor of language acquisition in young infants. Cognition, 29, 143 178.Molinaro, N., Lizarazu, M., Lallier, M., Bourguignon, M., \& Carreiras, M. (2016). Out-of-synchrony speech entrainment in developmental dyslexia. Human Brain Mapping, 37(8), 2767-2783.

Molinaro, N., \& Lizarazu, M. (2018). Delta (but not theta)-band cortical entrainment involves speechspecific processing. European Journal of Neuroscience, 48(7), 2642-2650.

Nichols, T. E., \& Holmes, A. P. (2002). Nonparametric permutation tests for functional neuroimaging: a primer with examples. Human Brain Mapping, 15(1), 1-25. 
Noordenbos, M. W., \& Serniclaes, W. (2015). The categorical perception deficit in dyslexia: A metaanalysis. Scientific Studies of Reading, 19(5), 340-359.

Obleser, J., Eisner, F., \& Kotz, S. A. (2008). Bilateral speech comprehension reflects differential sensitivity to spectral and temporal features. Journal of Neuroscience, 28(32), 8116-8123.

Poelmans, H., Luts, H., Vandermosten, M., Boets, B., Ghesquière, P., \& Wouters, J. (2012). Auditory steady state cortical responses indicate deviant phonemic-rate processing in adults with dyslexia. Ear and Hearing, 33(1), 134-143.

Poeppel, D. (2003). The analysis of speech in different temporal integration windows: cerebral lateralization as 'asymmetric sampling in time'. Speech Communication, 41(1), 245-255.

Power, A. J., Mead, N., Barnes, L., \& Goswami, U. (2013). Neural entrainment to rhythmic speech in children with developmental dyslexia. Frontiers in Human Neuroscience, 7, 777.

Power, A. J., Colling, L. J., Mead, N., Barnes, L., \& Goswami, U. (2016). Neural encoding of the speech envelope by children with developmental dyslexia. Brain and Language, 160, 1-10.

Ramus, F., Hauser, M. D., Miller, C., Morris, D., \& Mehler, J. (2000). Language discrimination by human newborns and by cotton-top tamarin monkeys. Science, 288, 349351.

Ramus, F. (2003). Developmental dyslexia: specific phonological deficit or general sensorimotor dysfunction?. Current Opinion in Neurobiology, 13(2), 212-218.

Ramus, F., \& Szenkovits, G. (2008). What phonological deficit?. The Quarterly Journal of Experimental Psychology, 61(1), 129-141.

Rosen, S. (2003). Auditory processing in dyslexia and specific language impairment: Is there a deficit? What is its nature? Does it explain anything?. Journal of Phonetics, 31(3-4), 509-527. 
Ramus, F., Altarelli, I., Jednorog, K., Zhao, J., \& di Covella, L. S. (2018). Neuroanatomy of developmental dyslexia: Pitfalls and promise. Neuroscience \& Biobehavioral Reviews, 84, 434-452.

Schroeder, C. E., \& Lakatos, P. (2009). Low-frequency neuronal oscillations as instruments of sensory selection. Trends in Neurosciences, 32(1), 9-18.

Taulu, S., Simola, J., \& Kajola, M. (2005). Applications of the signal space separation method. IEEE Transactions on Signal Processing, 53(9), 3359-3372.

Telkemeyer, S., Rossi, S., Koch, S. P., Nierhaus, T., Steinbrink, J., Poeppel, D., ... \& Wartenburger, I. (2009). Sensitivity of newborn auditory cortex to the temporal structure of sounds. Journal of Neuroscience, 29(47), 14726-14733.

Thwaites, A., Nimmo-Smith, I., Fonteneau, E., Patterson, R. D., Buttery, P., \& Marslen-Wilson, W. D. (2015). Tracking cortical entrainment in neural activity: auditory processes in human temporal cortex. Frontiers in Computational Neuroscience, 9, 5.

Wagner, R. K., \& Torgesen, J. K. (1987). The nature of phonological processing and its causal role in the acquisition of reading skills. Psychological Bulletin, 101(2), 192.

Wechsler, D. (2000). Manuel de l'Echelle d'Intelligence de Wechsler Pour Adultes-3e e'dition [Manual for the Wechsler Adult Intelligence Scale Third Edition]. Paris, France: ECPA.

Wechsler, D. (2008). Wechsler Adult Intelligence Scale WAIS-IV Canadian. Pearson. 


\section{Acknowledgments}

We thank to Prof. Christian Lorenzi (Laboratoire des systèmes perceptifs, Département d'études cognitives, Ecole normale supérieure) for designing the AM detection task. We also would like to acknowledge all the participants taking part in this study. This research was supported in part by the Agence Nationale de la Recherche (ANR-10-LABX-0087 IEC and ANR-10-IDEX-0001-02 PSL), the EUR Frontiers (ANR-17-EURE-0017), Fondation Pour l'Audition (FPA RD-2016-8 research grant).

\section{Data Availability Statement}

The data that support the findings of this study are available on request from the corresponding author. 


\section{Tables}

Table 1: Summary of the studies analyzing auditory neural entrainment in dyslexia. Abbreviations: EEG, electroencephalography; MEG, magnetoencephalography; fMRI, functional magnetic resonance imaging; fNIRS, functional near-infrared spectroscopy; SNR, signal-to-noise ratio; BOLD, blood-oxygen-level dependent; $\mathrm{N}_{\mathrm{C}}$, number of control participants; $\mathrm{N}_{\mathrm{D}}$, number of dyslexic participants; PLV, phase locking value; IHPS, inter-hemispheric phase synchronization; Hbo, oxygenated hemoglobin; HbR deoxyhemoglobin; NS, nonspeech; S, speech; D, dyslexic participants; C, control participants; ?, not analyzed; LH, left hemisphere; RH, right hemisphere; $d$, Cohen's $d$.

\begin{tabular}{|c|c|c|c|c|c|c|c|c|c|}
\hline Study & Technique & $N_{C}, N_{D}$ & $\begin{array}{l}\text { Age } \\
\text { (C, D) }\end{array}$ & Language & Stimuli & Measure & Delta & Theta & $\begin{array}{c}\text { Beta } \\
\text { Gamma }\end{array}$ \\
\hline $\begin{array}{l}\text { McAnally and } \\
\text { Stein, } 1997\end{array}$ & EEG & 15,15 & 27,28 & English & $\begin{array}{l}\text { AM white- } \\
\text { noise }\end{array}$ & SNR & ? & ? & $\begin{array}{c}D<C \\
d=0.56\end{array}$ \\
\hline $\begin{array}{c}\text { Menell et al., } \\
1999\end{array}$ & EEG & 21,24 & 26,28 & English & $\begin{array}{c}\text { AM white- } \\
\text { noise }\end{array}$ & SNR & ? & ? & $\begin{array}{c}D<C \\
d=0.47\end{array}$ \\
\hline $\begin{array}{l}\text { Lehongre et } \\
\text { al., } 2011\end{array}$ & MEG & 23,21 & 24,25 & French & $\begin{array}{l}\text { AM white- } \\
\text { noise }\end{array}$ & SNR & ? & ? & $\begin{array}{c}D<C \text { in } L H \\
d=?\end{array}$ \\
\hline $\begin{array}{l}\text { Poelmans et } \\
\text { al., } 2012\end{array}$ & EEG & 30,30 & 21,22 & Dutch & $\begin{array}{l}\text { AM speech } \\
\text { weighted- } \\
\text { noise }\end{array}$ & SNR & ? & $D=C$ & $\begin{array}{c}D<C \text { in } L H \\
d=0.52\end{array}$ \\
\hline $\begin{array}{l}\text { Lehongre et } \\
\text { al., } 2013\end{array}$ & fMRI/EEG & 15,17 & 24,24 & French & $\begin{array}{c}\text { Audiovisual } \\
\text { movie }\end{array}$ & $\begin{array}{l}\text { Correlation } \\
\text { between } \\
\text { BOLD and } \\
\text { EEG power }\end{array}$ & $D=C$ & $D=C$ & $\begin{array}{c}D<C \text { in } L H \\
d=0.71\end{array}$ \\
\hline $\begin{array}{c}\text { Power et al., } \\
2016\end{array}$ & EEG & 11,12 & 15,15 & English & $\begin{array}{c}\text { Noise vocoded } \\
\text { sentences }\end{array}$ & $\begin{array}{l}\text { Reconstructi } \\
\text { on Accuracy }\end{array}$ & $\begin{array}{c}D<C \\
d=0.81\end{array}$ & $D=C$ & ? \\
\hline
\end{tabular}




\begin{tabular}{|c|c|c|c|c|c|c|c|c|c|}
\hline $\begin{array}{l}\text { De Vos et al., } \\
2017\end{array}$ & EEG & 32,36 & 15,15 & Dutch & $\begin{array}{l}\text { AM white- } \\
\text { noise }\end{array}$ & SNR & ? & $D=C$ & $\begin{array}{c}D>C \\
d=-0.72\end{array}$ \\
\hline $\begin{array}{l}\text { Hämäläinen et } \\
\text { al., } 2012\end{array}$ & MEG & 10,11 & 28,22 & English & $\begin{array}{c}\text { AM white- } \\
\text { noise }\end{array}$ & PLV & $\begin{array}{c}D<C \text { in } R H \\
d=0.95\end{array}$ & $D=C$ & ? \\
\hline $\begin{array}{c}\text { Poelmans et } \\
\text { al., } 2012\end{array}$ & EEG & 30,30 & 21,22 & Dutch & $\begin{array}{l}\text { AM speech } \\
\text { weighted- } \\
\text { noise }\end{array}$ & IHPS & $?$ & $D=C$ & $\begin{array}{c}D<C \\
d=0.67\end{array}$ \\
\hline $\begin{array}{l}\text { Lizarazu et al., } \\
2015\end{array}$ & MEG & 42,42 & 20,23 & Spanish & $\begin{array}{c}\text { AM white- } \\
\text { noise }\end{array}$ & PLV & $D=C$ & $\begin{array}{c}D>C \\
d=-0.67\end{array}$ & $\begin{array}{c}\mathrm{D}>\mathrm{C} \text { in } \mathrm{RH} \\
\mathrm{d}=-0.78\end{array}$ \\
\hline $\begin{array}{c}\text { Cutini et al., } \\
2016\end{array}$ & fNIRS & 18,18 & 13,13 & English & $\begin{array}{c}\text { AM white- } \\
\text { noise }\end{array}$ & $\begin{array}{c}\mathrm{HbO} / \mathrm{HbR} \\
\text { concentratio } \\
\mathrm{n}\end{array}$ & $\begin{array}{c}D>C \text { in } R H \\
d=0.65\end{array}$ & $?$ & $D=C$ \\
\hline $\begin{array}{l}\text { Molinaro et } \\
\text { al., } 2016\end{array}$ & MEG & 20,20 & 20,23 & Spanish & Sentences & Coherence & $\begin{array}{c}D<C \text { in } R H \\
d=0.66\end{array}$ & $D=C$ & ? \\
\hline
\end{tabular}


Table 2: Testing predictions made by previous studies. Statistical results are marked in green if our results replicate previous studies' results and in red if our results do not replicate previous studies. Abbreviations : SNR, signal-to-noise ratio; PLV, phase locking value; IHPS, inter-hemispheric phase synchronization; D, dyslexic participants; $\mathrm{C}$, control participants; ?, not analyzed; $\mathrm{LH}$, left hemisphere; $\mathrm{RH}$, right hemisphere.

\begin{tabular}{|c|c|c|c|c|c|}
\hline Study & Stimuli & Measure & Delta & Theta & Gamma \\
\hline McAnally and Stein, 1997 & $\begin{array}{c}\text { AM white- } \\
\text { noise }\end{array}$ & SNR & $?$ & $?$ & $\begin{array}{c}D<C \\
t(76)=0.98, p=0.16, d=0.22\end{array}$ \\
\hline Menell et al., 1999 & $\begin{array}{c}\text { AM white- } \\
\text { noise }\end{array}$ & SNR & $?$ & $?$ & $\begin{array}{c}D<C \\
t(76)=0.98, p=0.16, d=0.22\end{array}$ \\
\hline Lehongre et al., 2011 & $\begin{array}{c}\text { AM white- } \\
\text { noise }\end{array}$ & SNR & $?$ & $?$ & $\begin{array}{c}D<C \text { in LH } \\
t(37)=0.87, p=0.39, d=0.28\end{array}$ \\
\hline Poelmans et al., 2012 & $\begin{array}{l}\text { AM white- } \\
\text { noise }\end{array}$ & SNR & $?$ & $\begin{array}{c}D=C \\
t(76)=1.41, p=0.14, d=0.31\end{array}$ & $\begin{array}{c}\text { D }<\text { C in LH } \\
t(37)=0.87, p=0.39, d=0.28\end{array}$ \\
\hline De Vos et al., 2017 & $\begin{array}{c}\text { AM white- } \\
\text { noise }\end{array}$ & SNR & $?$ & $\begin{array}{c}D<C \\
t(76)=1.41, p=0.07, d=0.31\end{array}$ & $\begin{array}{c}D>C \\
t(76)=0.98, p=0.84, d=0.22\end{array}$ \\
\hline Hämäläinen et al., 2012 & $\begin{array}{c}\text { AM white- } \\
\text { noise }\end{array}$ & PLV & $\begin{array}{c}D<C \text { in } R H \\
t(37)=-1.61, p=0.94, d=-0.52\end{array}$ & $\begin{array}{c}D=C \\
t(76)=-0.35, p=0.73, d=-0.08\end{array}$ & $?$ \\
\hline Poelmans et al., 2012 & $\begin{array}{c}\text { AM white- } \\
\text { noise }\end{array}$ & IHPS & $?$ & $\begin{array}{c}D=C \\
t(37)=-0.21, p=0.83, d=-0.07\end{array}$ & $\begin{array}{c}D<C \\
t(37)=0.33, p=0.37, d=0.11\end{array}$ \\
\hline Lizarazu et al., 2015 & $\begin{array}{l}\text { AM white- } \\
\text { noise }\end{array}$ & PLV & $\begin{array}{c}D=C \\
t(76)=-1.61, p=0.11, d=-0.37\end{array}$ & $\begin{array}{c}D>C \\
t(76)=-0.35, p=0.37, d=-0.08\end{array}$ & $\begin{array}{c}\text { D }>\text { C in RH } \\
t(37)=0.12, p=0.55, d=0.04\end{array}$ \\
\hline Molinaro et al., 2016 & Sentences & Coherence & $\begin{array}{c}D<C \text { in } R H \\
t(37)=-0.44, p=0.66, d=-0.14\end{array}$ & $\begin{array}{c}D=C \\
t(76)=1.4, p=0.17, d=0.32\end{array}$ & $?$ \\
\hline
\end{tabular}


Table 3: Correlation analysis. Correlations were computed between the behavioral scores and the brain measures showing significant group differences. Correlations were obtained for control (C), dyslexic (D) and all participants. We indicate the number of participants for each task $(\mathrm{N})$, the Pearson's correlation $(r)$ and the $p$-value (p). Significant correlations are highlighted in red.

\begin{tabular}{|c|c|c|c|c|c|c|}
\hline \multirow{2}{*}{$(\mathbf{N}, \mathbf{r}, \mathbf{p})$} & \multicolumn{2}{|c|}{ Ampl. $\boldsymbol{\rho}_{\max }$ at 2 HZ in RAC } & \multicolumn{2}{c|}{ Ampl. $\boldsymbol{\rho}_{\max }$ at 30 Hz in LAC } \\
\cline { 2 - 7 } & $\mathbf{C}$ & $\mathbf{D}$ & $\mathbf{C}$ and D & C & D & C and D \\
\hline Literacy index & $18,-0.19,0.45$ & $19,0.46,0.05$ & $37,0.3,0.07$ & $18,0.04,0.87$ & $19,-0.01,0.97$ & $37,0.39,0.02$ \\
\hline Phonological index & $18,-0.13,0.61$ & $19,0.44,0.06$ & $37,0.26,0.12$ & $18,-0.13,0.61$ & $19,0.44,0.05$ & $37,0.26,0.12$ \\
\hline RAN index & $18,-0.06,0.81$ & $18,-0.35,0.15$ & $36,-0.32,0.05$ & $18,-0.18,0.47$ & $18,0.11,0.66$ & $36,-0.28,0.1$ \\
\hline AM detection task & $16,-0.12,0.66$ & $16,-0.05,0.85$ & $32,-0.14,0.44$ & $16,0.13,0.63$ & $16,-0.27,0.31$ & $32,-0.11,0.55$ \\
\hline
\end{tabular}




\section{Figure Legends}

Figure 1: Phase cross-correlation analysis of the nonspeech stimuli. (A) Maximum phase correlation ( $\rho_{\max }$ ) values (mean and standard error) for the 2,5 and $30 \mathrm{~Hz} \mathrm{AM}$ white noise in left and the right auditory cortex in control (blue) and dyslexic (red) participants. (B) Time lag $(\tau)$ values of the phase $\rho_{\max }$ values for the 2, 5 and $30 \mathrm{~Hz}$ AM white noise in the left and right auditory cortex in control (blue) and dyslexic (red) participants. Bars and error bars indicate means and standard errors respectively. Each dot represents the data of each participant and the shaded area is the data distribution.

Figure 2: Amplitude cross-correlation analysis of the nonspeech stimuli. (A) Maximum amplitude correlation ( $\rho_{\max }$ ) values (mean and standard error) for the 2, 5 and $30 \mathrm{~Hz}$ AM white noise in the left and the right auditory cortex in control (blue) and dyslexic (red) participants. (B) Time lag $(\tau)$ values of the amplitude $\rho_{\max }$ values for the 2,5 and $30 \mathrm{~Hz}$ AM white noise in the left and the right auditory cortex in control (blue) and dyslexic (red) participants. Bars and error bars indicate means and standard errors respectively. Each dot represents the data of each participant and the shaded area is the data distribution.

Figure 3: Phase cross-correlation analysis of the speech stimuli. Maximum amplitude correlation ( $\left.\rho_{\max }\right)$ values (mean and standard error) for the forward (A) and backward (B) speech in delta and theta bands in the left and right auditory cortex in control (blue) and dyslexic (red) participants. Bars and error bars indicate means and standard errors respectively. Each dot represents the data of each participant and the shaded area is the data distribution.

Figure 4: Amplitude cross-correlation analysis of the speech stimuli. Maximum amplitude correlation $\left(\rho_{\max }\right)$ values (mean and standard error) for the forward $(A)$ and backward $(B)$ speech in delta, theta, alpha, beta and gamma bands in the left and right auditory cortex in control (blue) and dyslexic (red) participants. 
Bars and error bars indicate means and standard errors respectively. Each dot represents the data of each participant and the shaded area is the data distribution.

Figure 5: Correlation plots. (A) Correlation between the amplitude cross-correlation values at $2 \mathrm{~Hz}$ in the right auditory cortex (RAC) and the literacy index. (B) Correlation between the amplitude cross-correlation values at $30 \mathrm{~Hz}$ in the left auditory cortex (LAC) and the literacy index. (C) Correlation between the amplitude cross-correlation values obtained at $30 \mathrm{~Hz}$ in the LAC and the phonological index. (D) Correlation between the amplitude cross-correlation values obtained at $2 \mathrm{~Hz}$ in the RAC and the rapid automatized naming (RAN) index. Blue dots represent the values for the control participants; red dots represent values for the dyslexic participants. Pearson's correlations $(r)$ and regression lines are shown for all participants (black line) and for each group separately (blue, control participants; red, dyslexic participants). 
Figures

\section{Figure 1:}

A

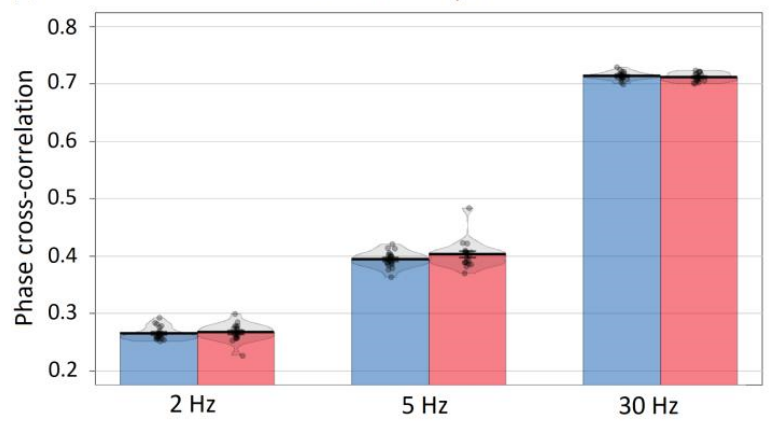

Left auditory cortex

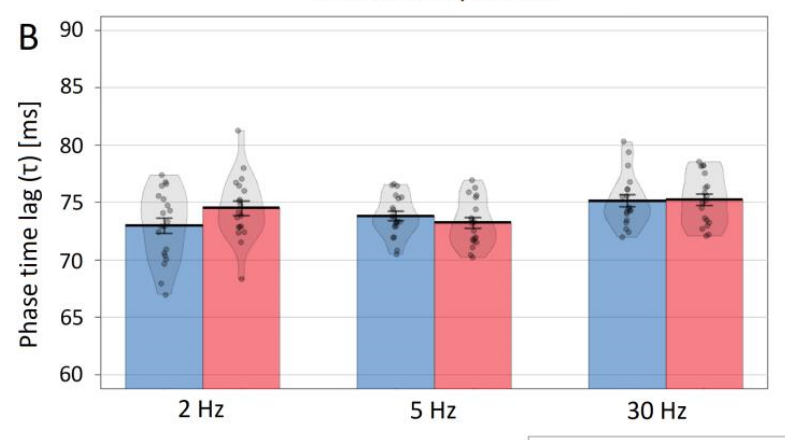

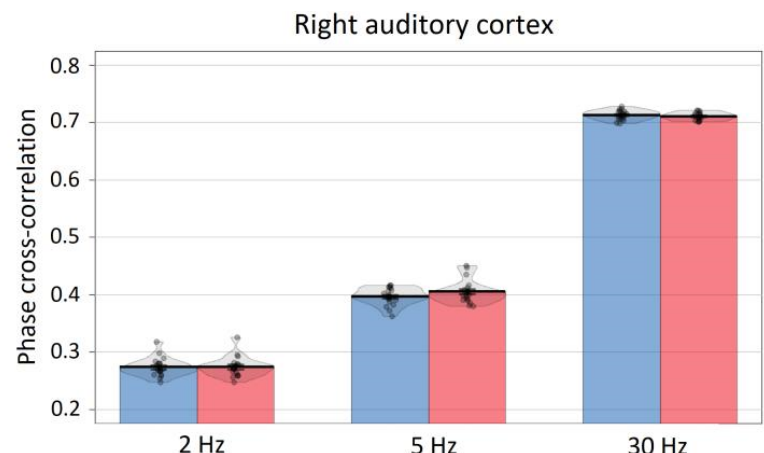

$5 \mathrm{~Hz}$

$30 \mathrm{~Hz}$

Right auditory cortex

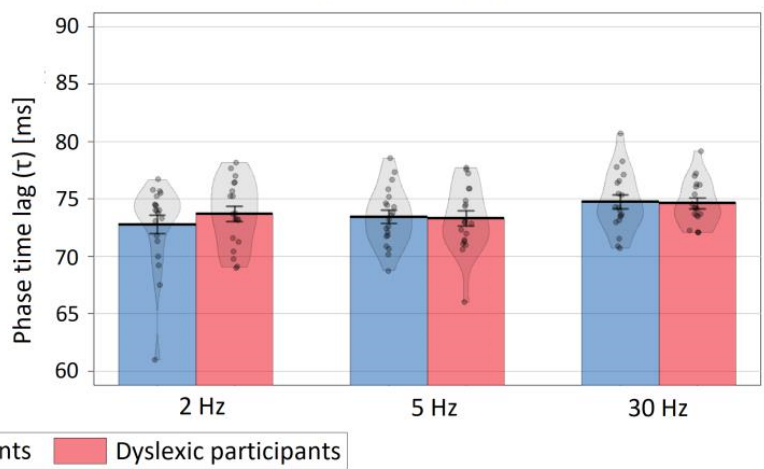

\section{Figure 2:}

A

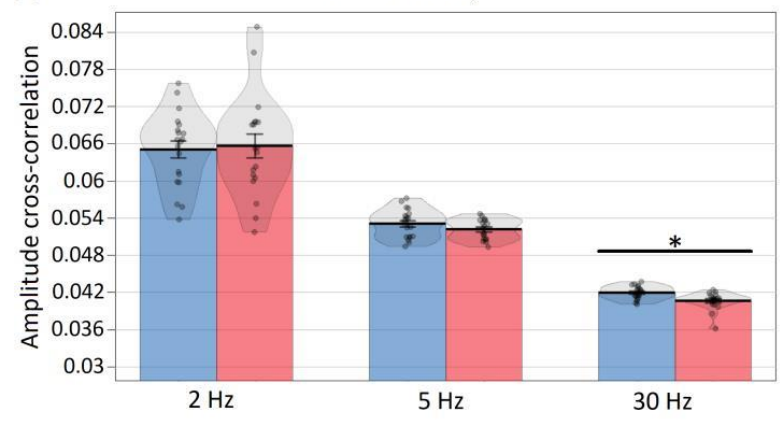

B

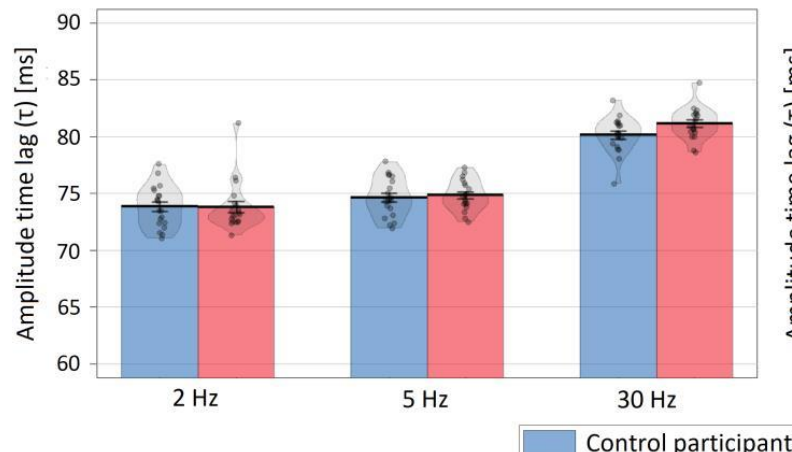

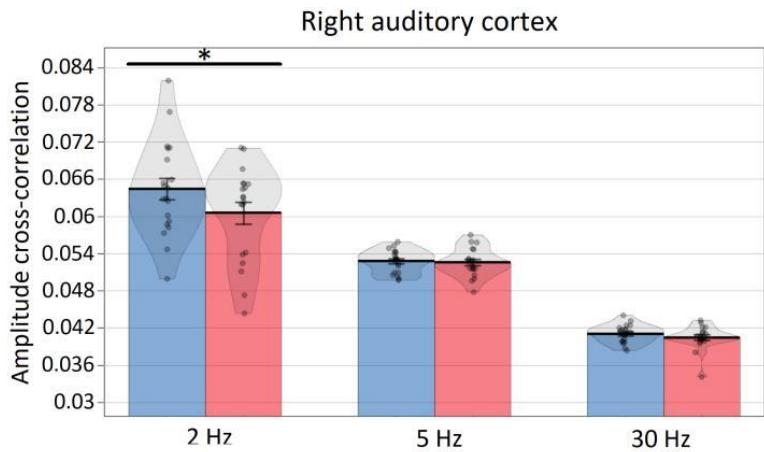

$5 \mathrm{~Hz}$

Right auditory cortex






\section{Figure 3:}

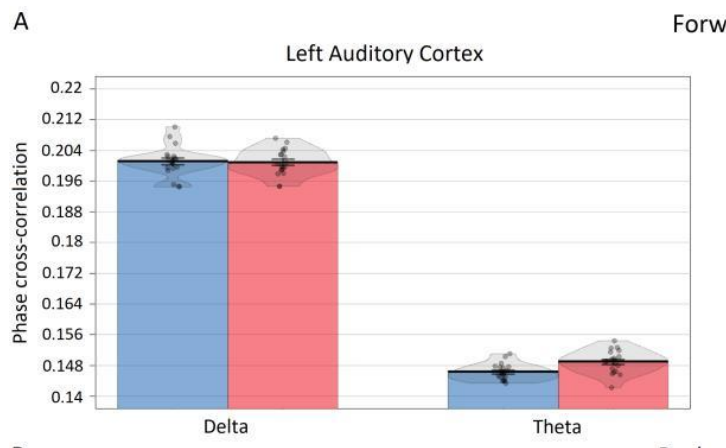

Forward speech

B

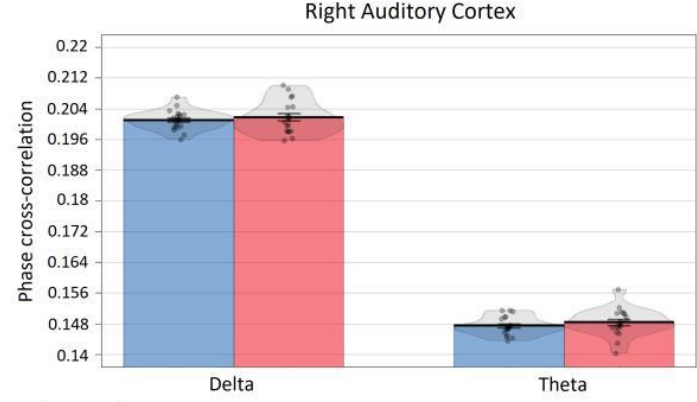

Left Auditory Cortex

Backward speech
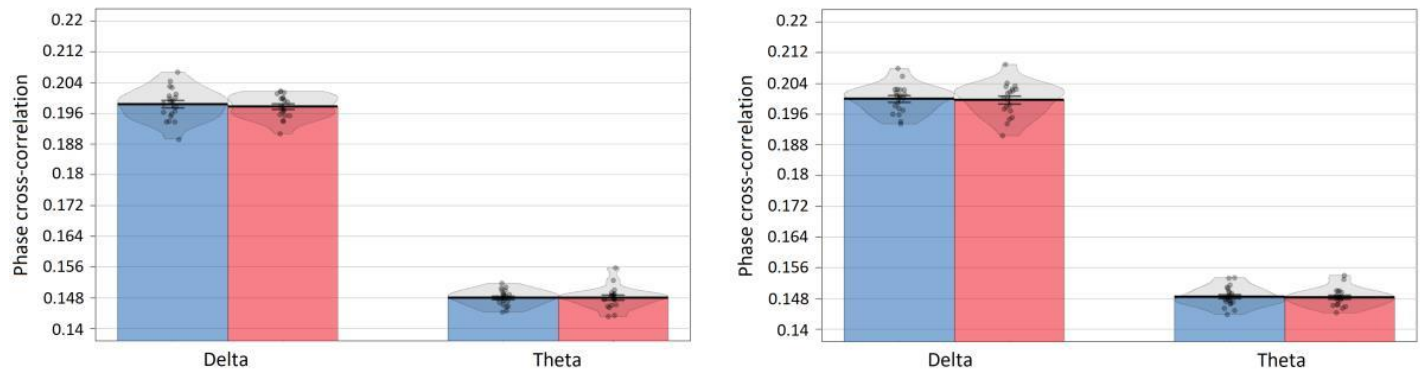

Control participants $\square$ Dyslexic participants

\section{Figure 4:}

$A$

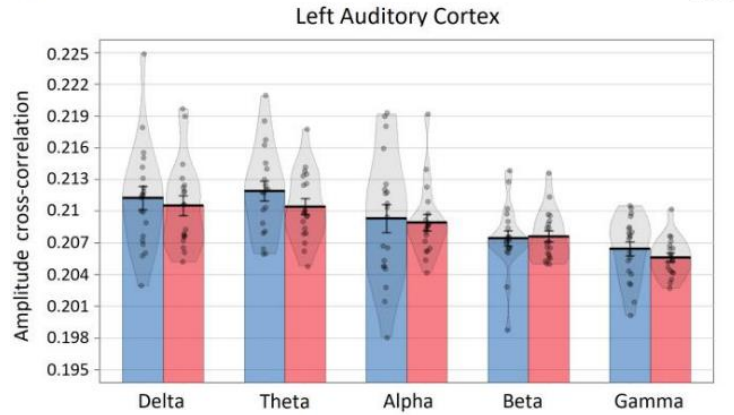

Forward speech

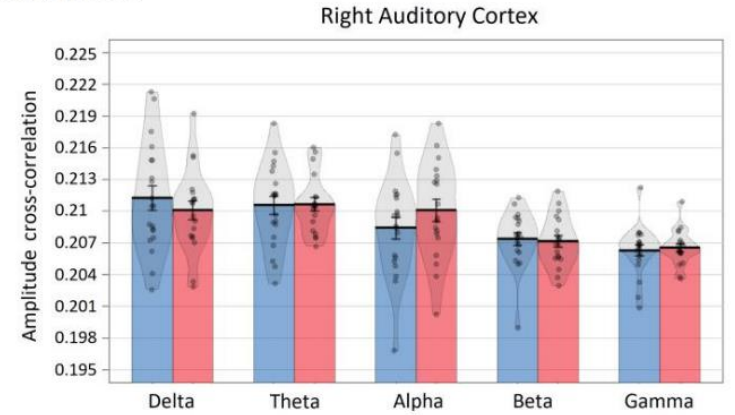

B

Left Auditory Cortex
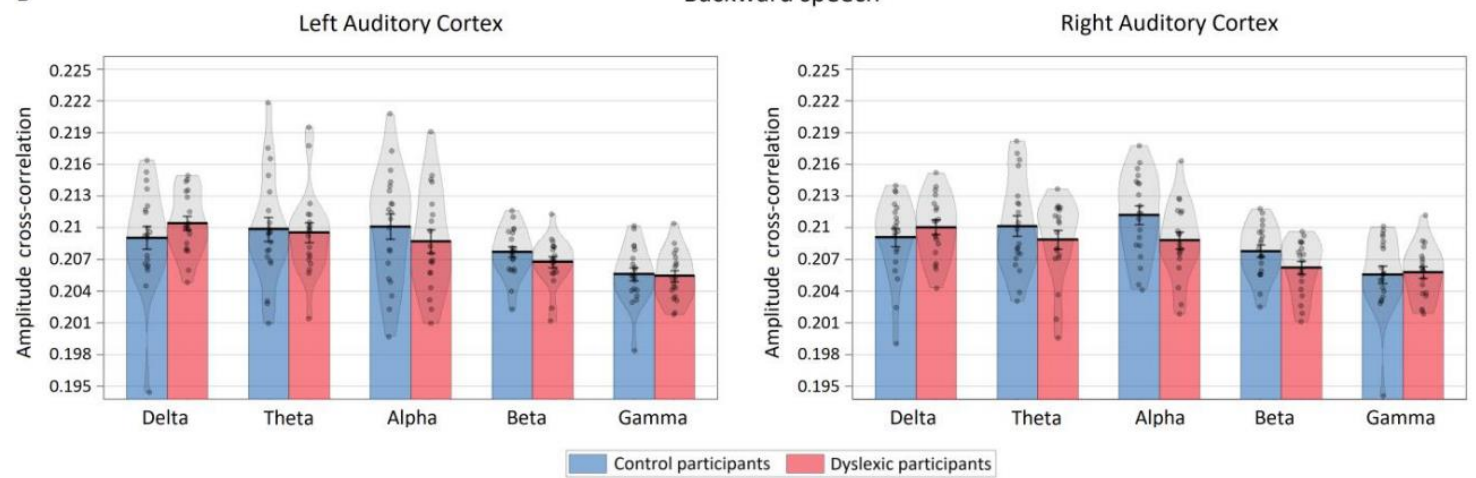
Figure 5:

A

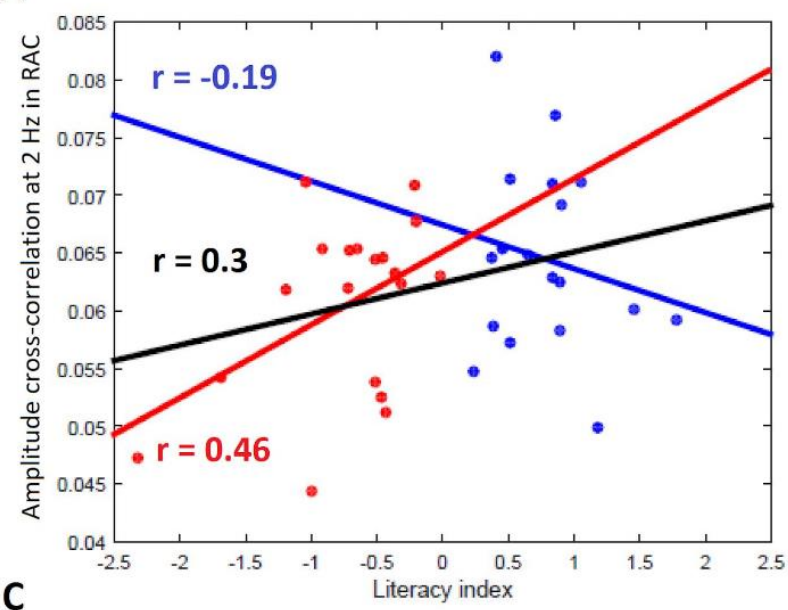

C

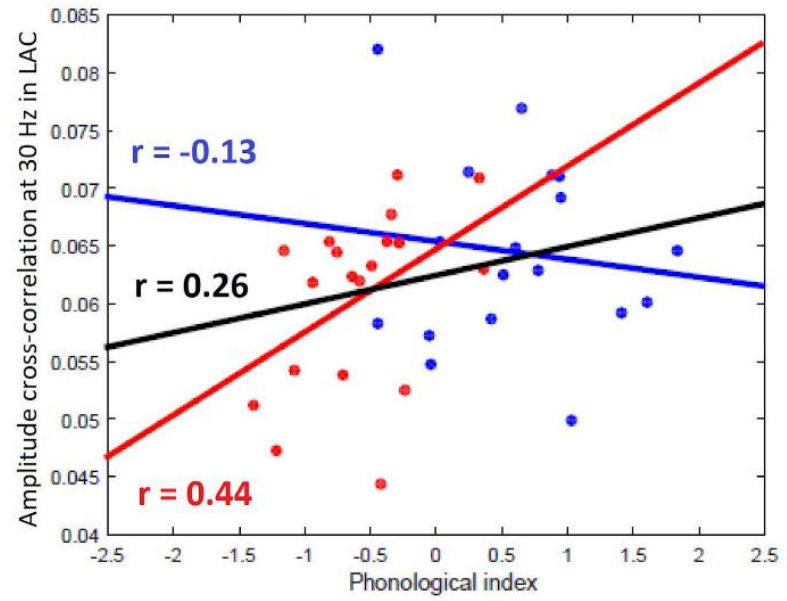

B
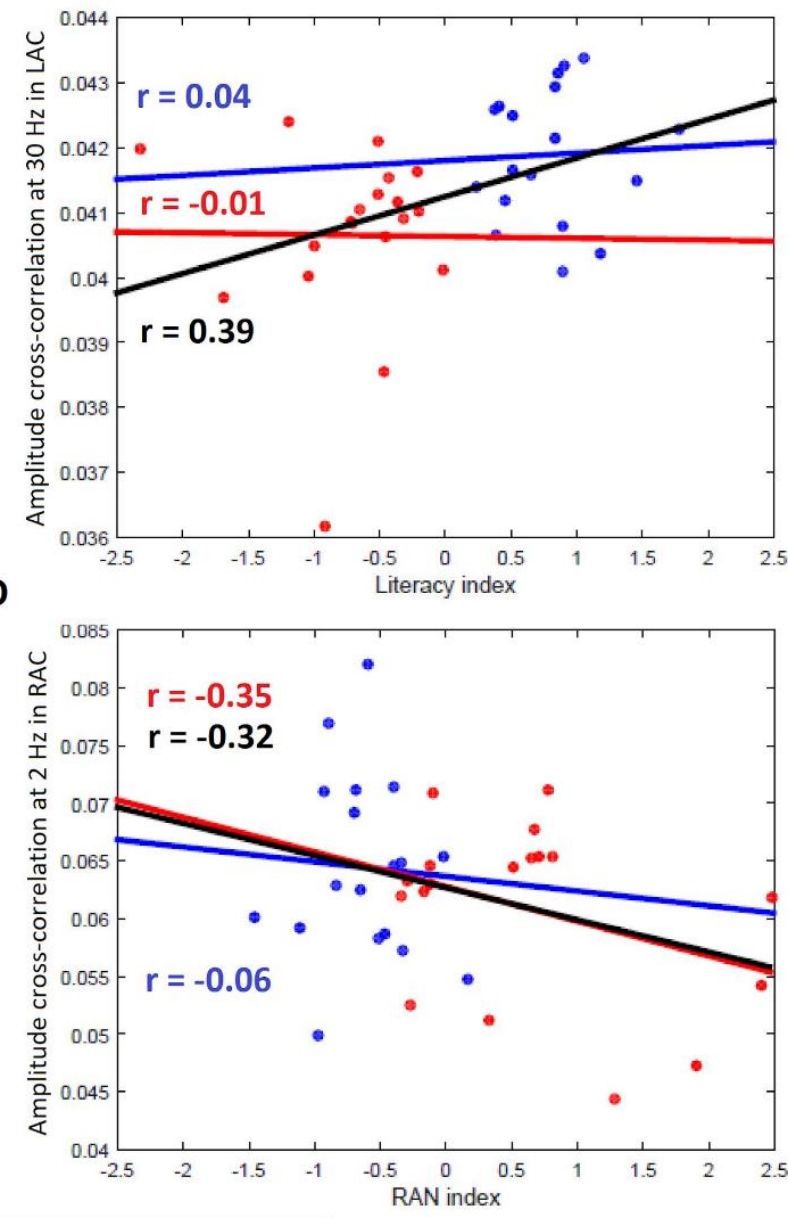


\section{Supplementary material}

\section{Supplementary Figures}

Supplementary Figure 1: Localization of auditory regions. (A) MEG auditory evoked response to the stationary white noise condition. (B) Auditory evoked fields in the MEG sensor space in the $0.1-0.3 \mathrm{~s}$ (pre-stimulus) interval compared to the $-0.2-0$ (post-stimulus) interval in left (LH) and right (RH) hemisphere. Sensors showing significant response in the post- compared to the pre-interval are highlighted. (C) Source estimates evoked by the stationary white noise. These regions were then defined as Regions Of Interest (ROIs). The ROIs contained left and right auditory regions and mainly overlapped with Brodmann area 41 (BA41) and 42 (BA42) (primary auditory regions). The brain slice in the axial plane at $Z=18$ (MNI coordinates) illustrates source depth.

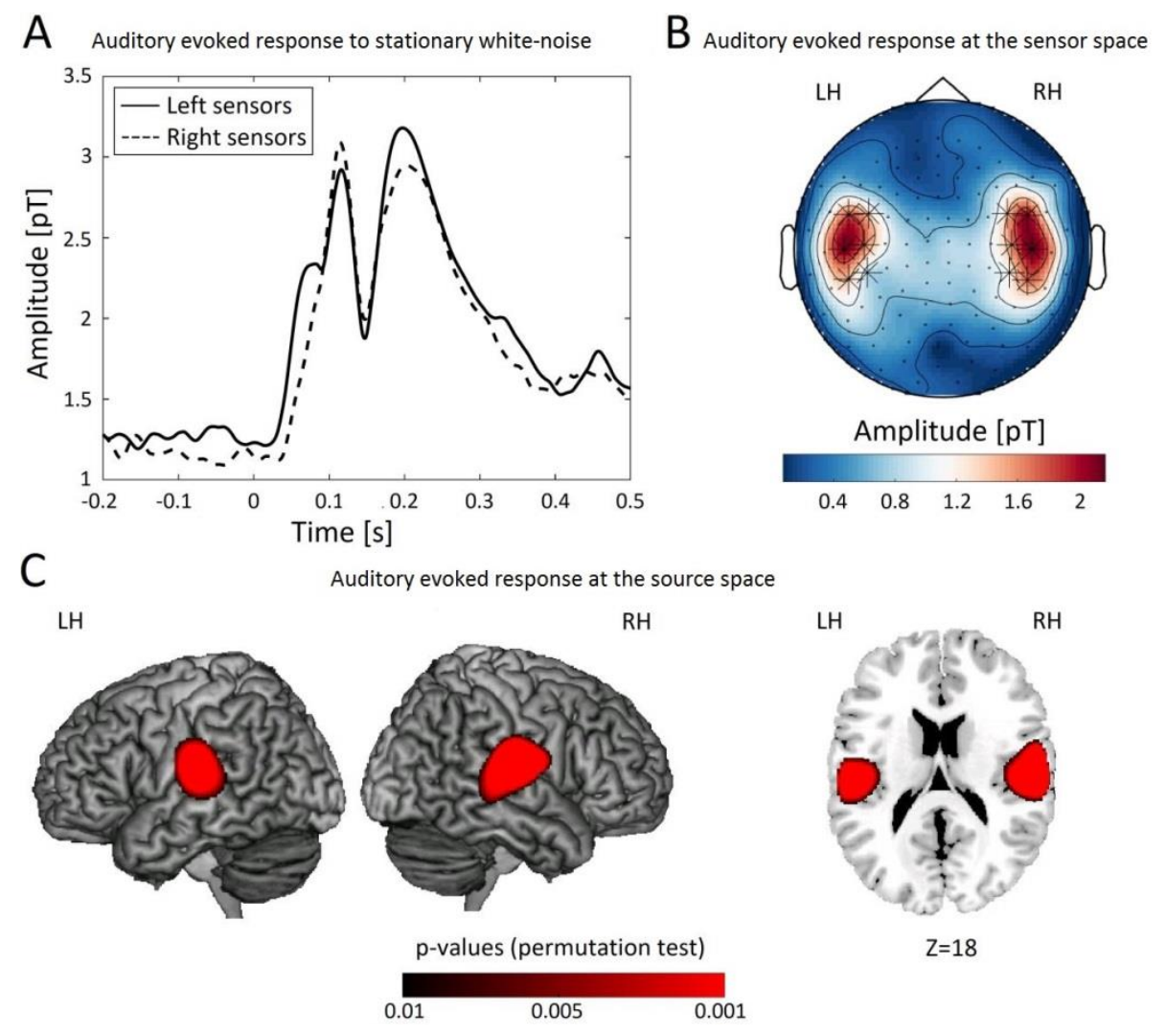


Supplementary Figure 2: Spectrogram of the auditory-steady state response (ASSR) to nonspeech stimuli. Power of the ASSR was estimated measuring the signal to noise ratio (SNR) of cortical evoked fields. We represented the SNR spectrogram $(0-40 \mathrm{~Hz})$ for the $2(\mathrm{~A}), 5(\mathrm{~B})$ and $30 \mathrm{~Hz}(\mathrm{C})$ amplitude modulated (AM) white noise in the left and the right auditory cortex in all participants. Shaded area represents the standard error.
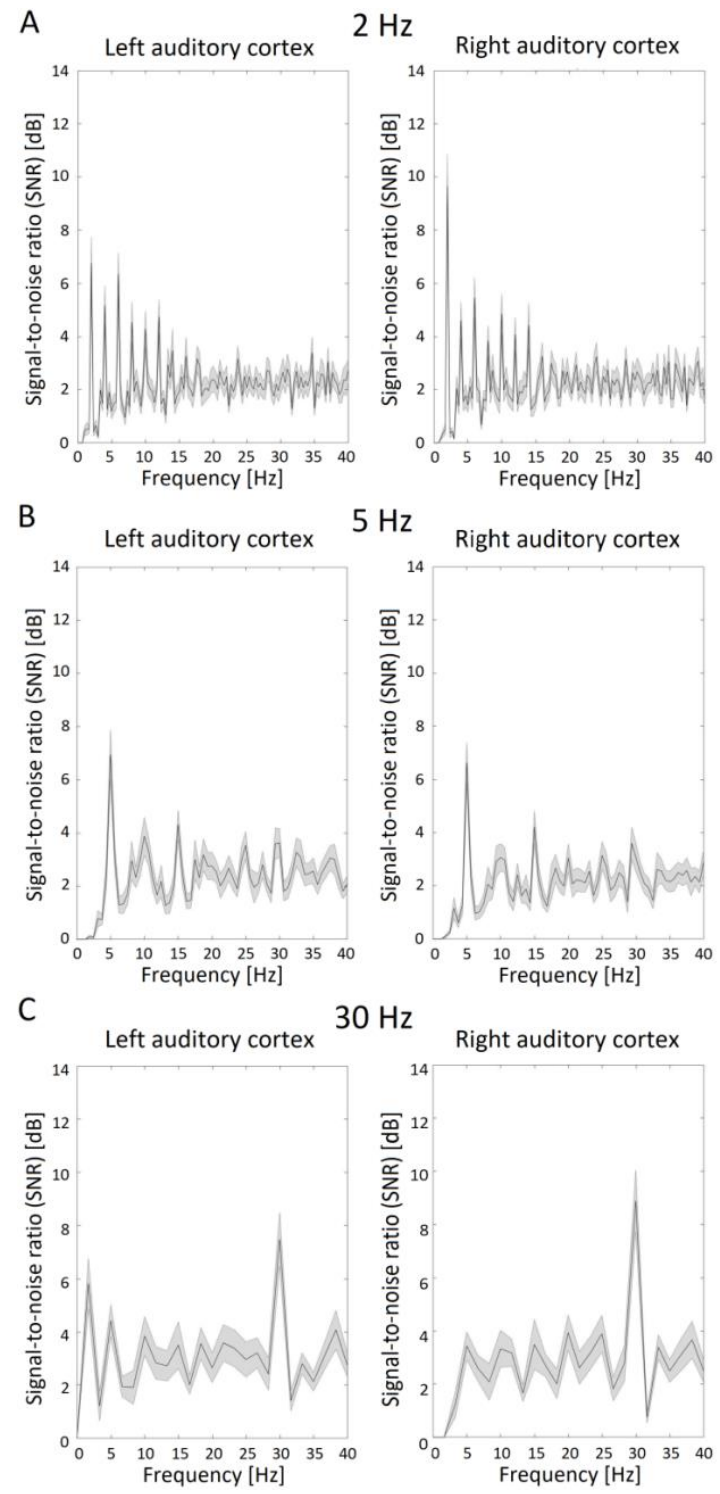
Supplementary Figure 3: Power and phase of the auditory steady-state responses (ASSR) to the nonspeech stimuli. (A) Power of the ASSR to 2, 5 and $30 \mathrm{~Hz}$ amplitude modulation (AM) was estimated based on the signal-to-noise ratio (SNR). (B) Phase locking value (PLV) of the auditory ASSR to 2, 5 and 30 $\mathrm{Hz}$ amplitude modulation (AM). Bars and error bars indicate means and standard errors respectively, in left and right auditory cortex, in control (blue) and dyslexic (red) participants. Each dot represents the data of each participant and the shaded area represents the data distribution.

A



B

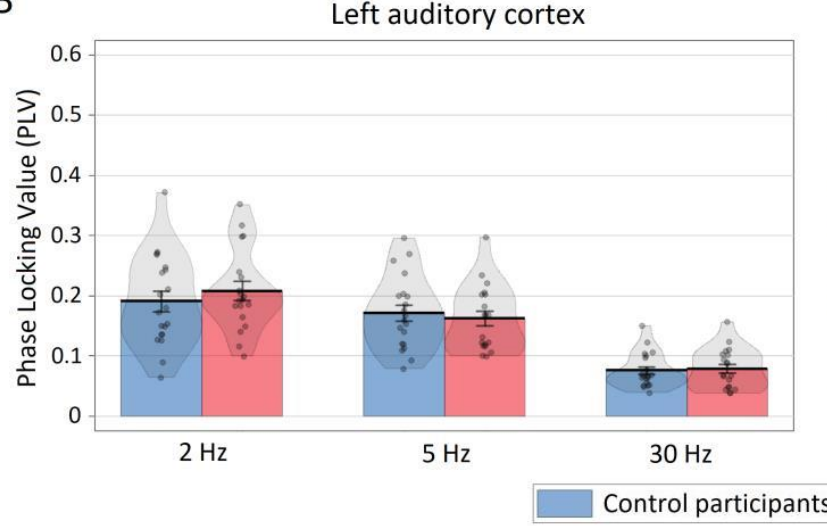

Right auditory cortex

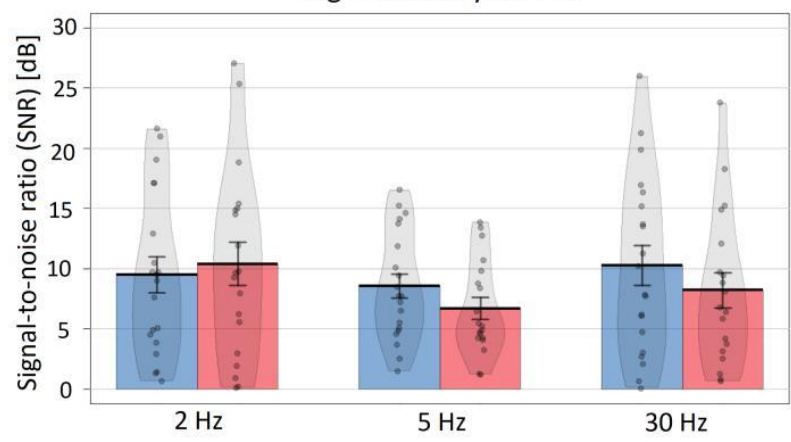

Right auditory cortex

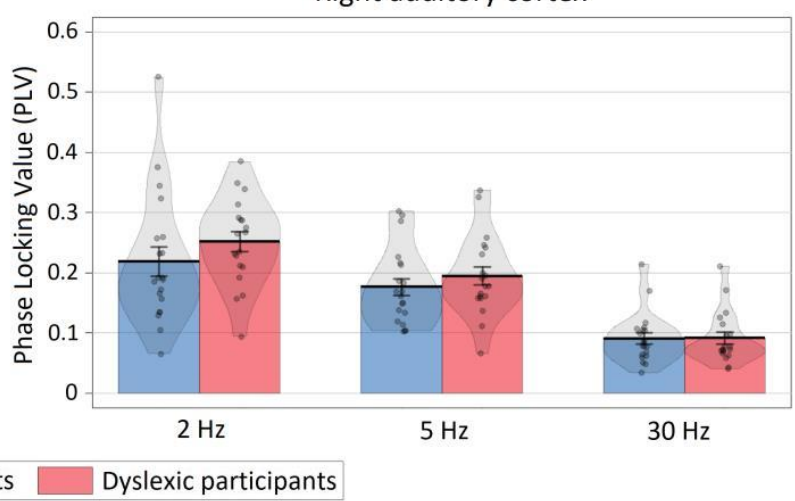


Supplementary Figure 4: Spectral profile of Coherence values in the $0.5-15 \mathrm{~Hz}$ frequency band for forward speech in the left and right auditory regions in all participants. Horizontal black lines show 95th percentile of chance distribution of the coherence values obtained from shuffled surrogate data. We observed significant higher coherence values (forward speech > surrogate data) in the delta $(0.5-2.5 \mathrm{~Hz})$ and theta $(3.5-6 \mathrm{~Hz})$ bands in left and right auditory regions. Shaded area represents the standard error.
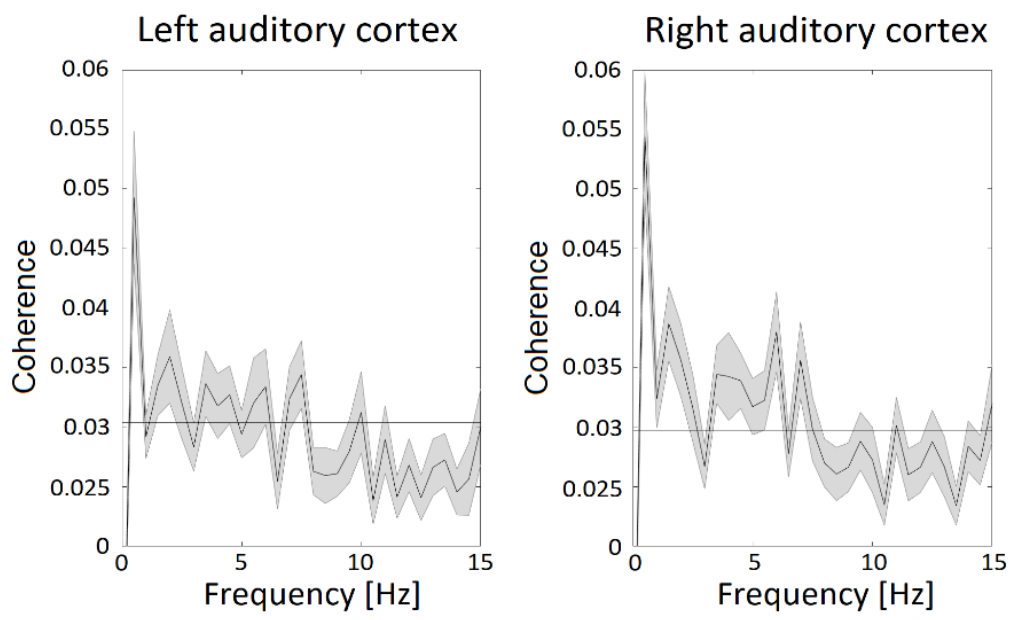
Supplementary Figure 5: Coherence analysis for the forward and backward speech stimuli. Coherence values for the forward (A) and the backward (B) speech in delta $(0.5-2.5 \mathrm{~Hz})$ and theta $(3.5-6 \mathrm{~Hz})$ frequency bands in left and the right auditory cortex in control (blue) and dyslexic (red) participants. Bars and error bars indicate means and standard errors respectively. Each dot represents the value of each participant and the shaded area is the data distribution.

A

Forward speech
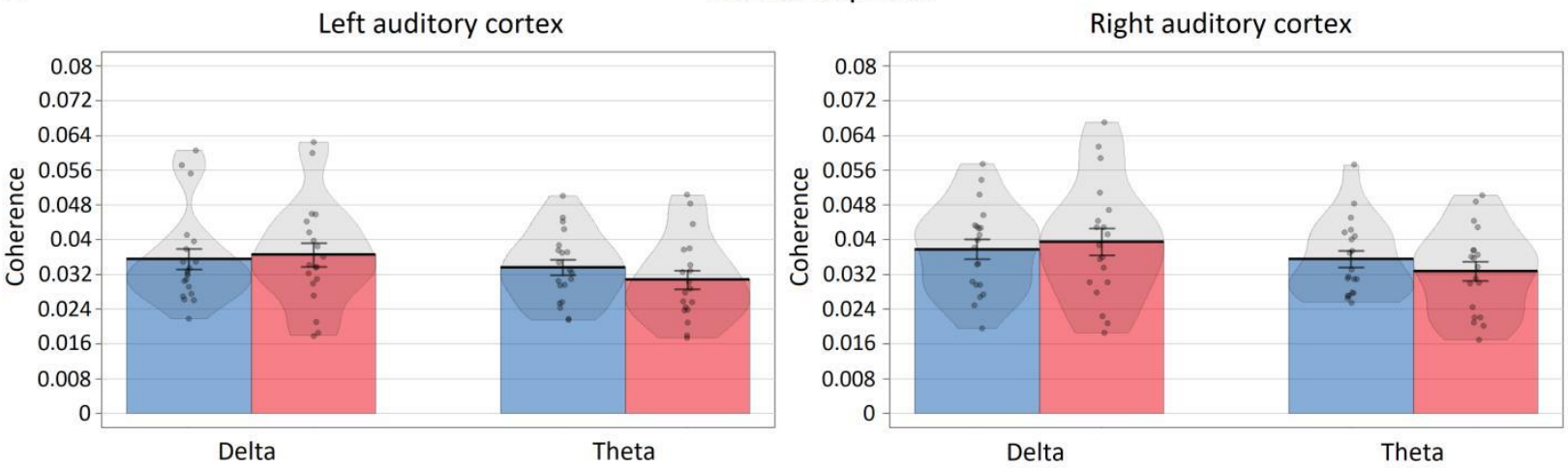

B

Backward speech

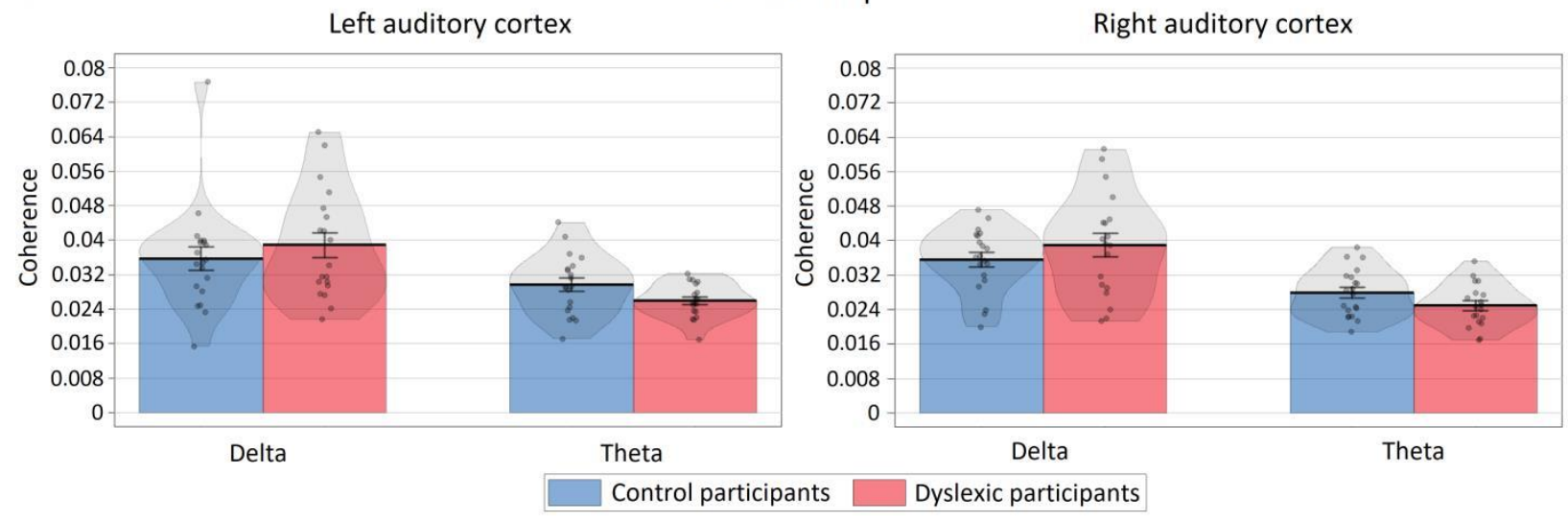


Supplementary Figure 6: Spectral profile of the phase (A) and amplitude (B) correlation $\left(\rho_{\max }\right)$ values for the $2 \mathrm{~Hz}$ (continuous line), $5 \mathrm{~Hz}$ (dashed line) and $30 \mathrm{~Hz}$ (dotted line) amplitude modulated (AM) white noise in the left and the right auditory cortex for all participants. Shaded area represents the standard error.
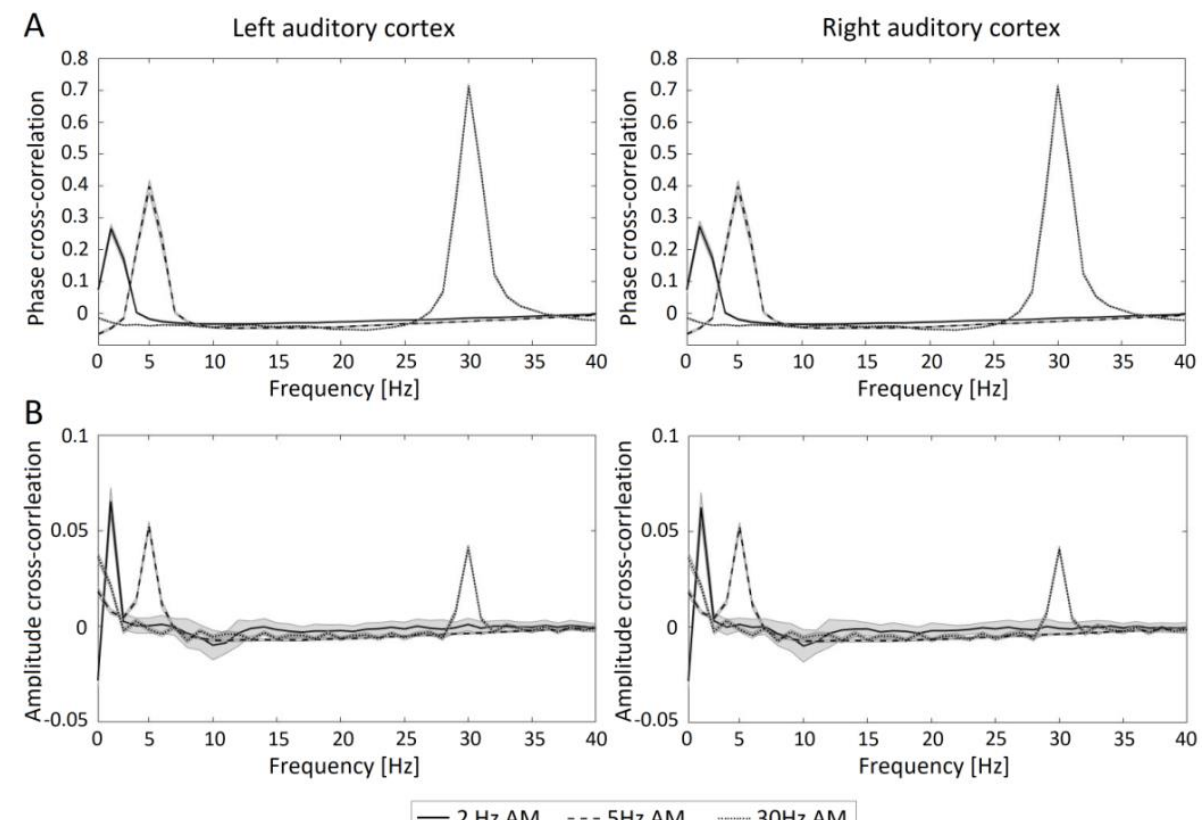

$-2 \mathrm{~Hz}$ AM $-\cdots 5 \mathrm{~Hz}$ AM $-30 \mathrm{~Hz}$ AM 
Supplementary Figure 7: Spectral profile of the phase (A) and amplitude (B) correlation $\left(\rho_{\max }\right)$ values for forward speech in the left and right auditory cortex for all participants. Horizontal black lines show 95th percentile of chance distribution of the phase and amplitude $\rho_{\max }$ values obtained from shuffled surrogate data. Shaded area represents the standard error. We observed significant (forward > surrogate) phase $\rho_{\max }$ values in the delta $(1-3 \mathrm{~Hz})$ and theta $(4-8 \mathrm{~Hz})$ bands. We observed significant (forward > surrogate) amplitude $\rho_{\max }$ values in the delta $(1-3 \mathrm{~Hz})$, theta $(4-8 \mathrm{~Hz})$, alpha $(9-12 \mathrm{~Hz})$, beta $(15-25 \mathrm{~Hz})$ and gamma (35 - $40 \mathrm{~Hz}$ ) bands.
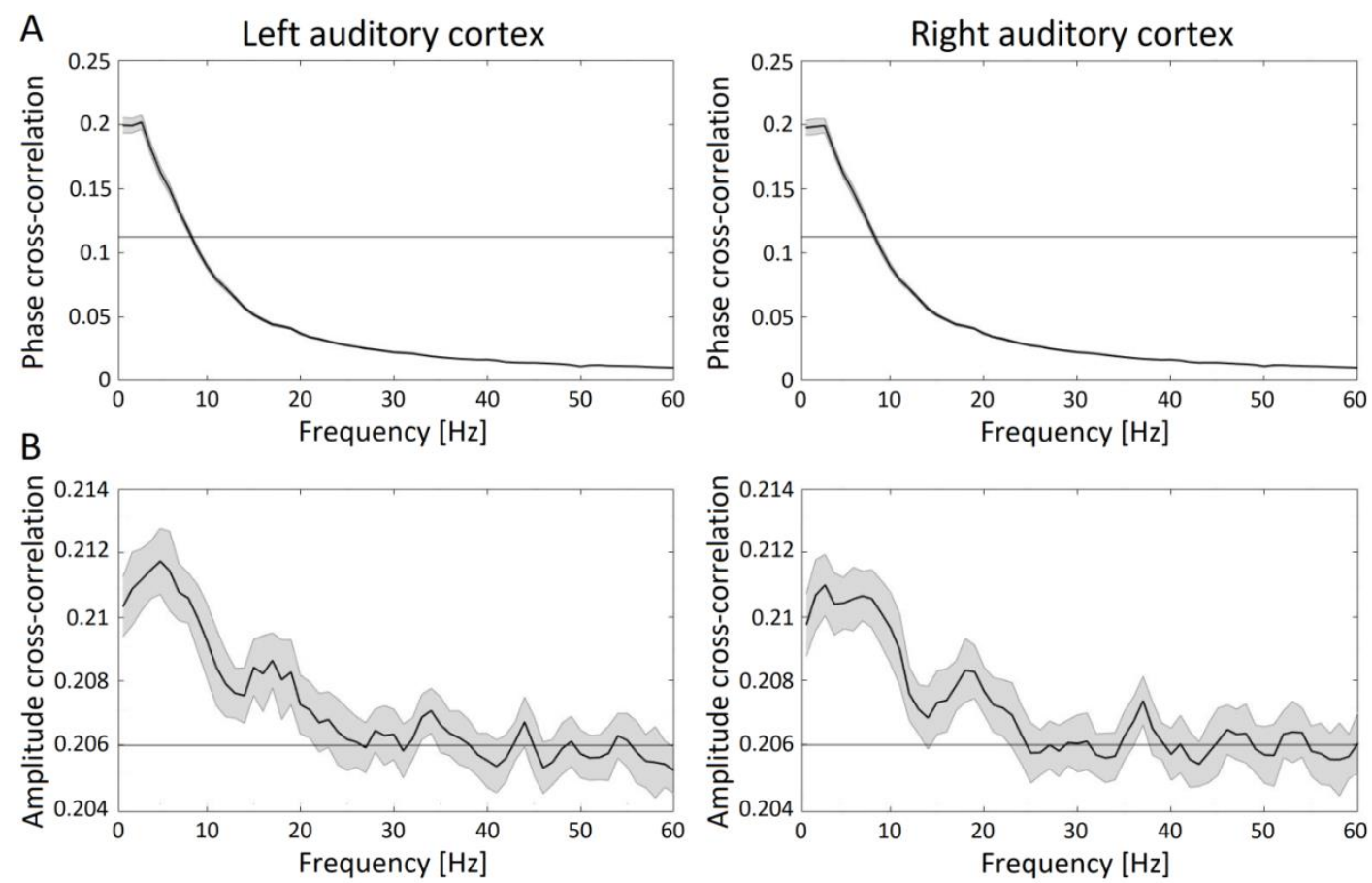


\section{Supplementary Tables}

Supplementary Table 1: Behavioral assessment for control and dyslexic participants. Characteristics of the two groups of participants regarding their IQ, reading, orthographic, phonological, musical skills and AM detection task. For each measurement, control and dyslexic participants were compared using an independent t-test (2-tails) (the t-value (t), the p-value (p), the degrees of freedom (df) and the Cohen's d (d) values are reported). Abbreviations: N, Number of participants; SD, Standard Deviation; IQ, Intelligence Quotient; CRWM, Correctly Read Words per Minute; RAN, Rapid Automatized Naming; Am, Amplitude Modulation.

\begin{tabular}{|c|c|c|c|c|c|}
\hline & \multicolumn{2}{|c|}{$\begin{array}{c}\text { Control } \\
\text { participants }\end{array}$} & \multicolumn{2}{|c|}{$\begin{array}{c}\text { Dyslexic } \\
\text { participants }\end{array}$} & \multirow{2}{*}{$\begin{array}{l}\text { Control vs. Dyslexic } \\
\text { participants } \\
\text { t(df), p, Cohen's d }\end{array}$} \\
\hline & $\mathbf{N}$ & Mean (SD) & $\mathbf{N}$ & Mean (SD) & \\
\hline \multicolumn{6}{|l|}{ IQ } \\
\hline Verbal & 16 & $122.31(16.03)$ & 18 & $104.88(15.34)$ & $t(32)=2.95, p<0.05, d=1.02$ \\
\hline Non-verbal & 17 & $112.41(12.87)$ & 18 & $106.94(14.78)$ & $\mathrm{t}(33)=1.16, p=0.25, \mathrm{~d}=0.39$ \\
\hline \multicolumn{6}{|l|}{ Reading skills } \\
\hline L'Alouette text (CRWM) & 18 & $178.14(27.26)$ & 19 & $95.41(30.11)$ & $t(35)=8.75, p<0.01, d=2.88$ \\
\hline L'Alouette text (z-score) & 18 & $0.74(1.04)$ & 19 & $-2.41(1.15)$ & $\mathrm{t}(35)=8.75, p<0.01, d=2.88$ \\
\hline Le Pollueur text (CRWM) & 17 & $198.35(20.72)$ & 19 & $125.01(34.44)$ & $t(34)=7.63, p<0.01, d=2.55$ \\
\hline Le Pollueur text (z-score) & 17 & $0.58(0.81)$ & 19 & $-2.31(1.35)$ & $t(34)=7.63, p<0.01, d=2.55$ \\
\hline \multicolumn{6}{|l|}{ Word reading (z-scores) } \\
\hline - Regular words & 17 & $-0.09(1.15)$ & 18 & $-2.08(0.70)$ & $t(33)=6.24, p<0.01, d=2.1$ \\
\hline - $\quad$ Non-regular words & 17 & $-0.15(0.91)$ & 19 & $-1.56(0.75)$ & $t(34)=5.07, p<0.01, d=1.69$ \\
\hline - Pseudo-words & 17 & $0.04(1.10)$ & 19 & $-2.31(0.61)$ & $\mathrm{t}(34)=7.98, p<0.01, d=2.66$ \\
\hline \multicolumn{6}{|l|}{ Orthographic skills } \\
\hline \multicolumn{6}{|l|}{ Word dictation } \\
\hline $\begin{array}{l}\text { Regular words accuracy (z- } \\
\text { score) }\end{array}$ & 17 & $1.05(0.35)$ & 19 & $-0.73(1.33)$ & $t(34)=5.35, p<0.01, d=1.79$ \\
\hline - $\quad$ Regular words time (s) & 17 & $35.01(6.46)$ & 19 & $65.84(42.65)$ & $t(34)=-2.95, p<0.01, d=-0.98$ \\
\hline $\begin{array}{l}\text { - Irregular words accuracy } \\
\text { (z-score) }\end{array}$ & 17 & $1.65(0.93)$ & 19 & $-1.21(1.24)$ & $t(34)=7.74, p<0.01, d=2.58$ \\
\hline - Irregular words time (s) & 17 & $41.82(8.41)$ & 19 & $69.11(42.42)$ & $t(34)=-2.6, p=0.01, d=-0.87$ \\
\hline
\end{tabular}




\begin{tabular}{|c|c|c|c|c|c|}
\hline $\begin{array}{l}\text { - Pseudo-words accuracy (z- } \\
\text { score) }\end{array}$ & 17 & $9.47(0.81)$ & 19 & $8.12(3.67)$ & $t(34)=1.6, p=0.12, d=0.54$ \\
\hline - $\quad$ Pseudo-words time (s) & 17 & $45.70(7.02)$ & 19 & $74.42(50.26)$ & $t(34)=-2.33, p=0.03, d=-0.78$ \\
\hline \multicolumn{6}{|l|}{ Text dictation (z-scores) } \\
\hline - Spelling accuracy & 17 & $0.93(0.41)$ & 19 & $-1.79(1.78)$ & $t(34)=6.14, p<0.01, d=2.05$ \\
\hline - Grammar accuracy & 17 & $0.94(0.68)$ & 19 & $-1.37(1.54)$ & $t(34)=5.7, p<0.01, d=1.9$ \\
\hline $\begin{array}{l}\text { Orthographic choice (items } \\
\text { correct per second) }\end{array}$ & 17 & $0.33(0.16)$ & 19 & $0.10(0.04)$ & $t(34)=5.95, p<0.01, d=1.98$ \\
\hline \multicolumn{6}{|l|}{ Phonological skills } \\
\hline \multicolumn{6}{|l|}{$\begin{array}{l}\text { Phonological } \\
\text { memory }\end{array}$} \\
\hline Digit-Span (scaled score) & 17 & $12.06(2.54)$ & 19 & $6.74(2.79)$ & $\mathrm{t}(34)=5.97 \mathrm{t}, \mathrm{p}<0.01, \mathrm{~d}=1.99$ \\
\hline Digit-Span forward (z-score) & 16 & $0.93(0.75)$ & 19 & $-0.60(0.91)$ & $t(33)=5.34, p<0.01, d=1.81$ \\
\hline Digit-Span backward (z-score) & 16 & $0.85(1.11)$ & 19 & $-0.26(0.76)$ & $\mathrm{t}(33)=3.49, \mathrm{p}<0.01, \mathrm{~d}=1.19$ \\
\hline \multicolumn{6}{|l|}{ Pseudo-word repetition } \\
\hline 5 syllables & 17 & $0.85(0.14)$ & 19 & $0.41(0.31)$ & $t(34)=5.26, p<0.01, d=1.76$ \\
\hline 7 syllables & 17 & $0.16(0.15)$ & 19 & $0.04(0.10)$ & $t(34)=2.83, p<0.01, d=0.95$ \\
\hline \multicolumn{6}{|l|}{ Phonological awareness } \\
\hline \begin{tabular}{ll} 
Phonemic & \multicolumn{2}{c}{ deletion } \\
(number correct per \\
second)
\end{tabular} & 17 & $0.45(0.28)$ & 19 & $0.21(0.13)$ & $t(34)=3.35, p<0.01, d=1.12$ \\
\hline $\begin{array}{l}\text { - Spoonerisms (number } \\
\text { correct per second) }\end{array}$ & 17 & $0.14(0.07)$ & 19 & $0.04(0.04)$ & $t(34)=5.01, p<0.01, d=1.67$ \\
\hline \multicolumn{6}{|l|}{ Rapid automatized naming } \\
\hline - $\quad$ RAN Objects (s) & 18 & $63.22(7.65)$ & 18 & $89.15(21.52)$ & $t(34)=-4.81, p<0.01, d=-1.6$ \\
\hline - $\quad$ RAN Digits (s) & 18 & $32.89(7.27)$ & 18 & $52.37(15.83)$ & $t(34)=-4.74, p<0.01, d=-1.58$ \\
\hline - $\quad$ RAN Colors (s) & 18 & $54.50(9.24)$ & 18 & 76.10(18.01) & $t(34)=-4.53, p<0.01, d=-1.51$ \\
\hline Musical practice (years) & 17 & $6.47(6.74)$ & 18 & $3.02(4.71)$ & $t(33)=1.77, p=0.08, d=0.6$ \\
\hline \multicolumn{6}{|l|}{$\begin{array}{l}\text { AM detection task } \\
\text { (modulation depth, \%) }\end{array}$} \\
\hline - $\quad$ AM_4Hz & 16 & $14.26(5.11)$ & 17 & $17.67(6.61)$ & $t(30)=-1.67, p=0.11, d=-0.58$ \\
\hline - $\quad$ AM_32Hz & 17 & $8.68(1.56)$ & 17 & $8.80(1.93)$ & $t(31)=-0.2, p=0.84, d=-0.07$ \\
\hline - $\quad$ AM_64Hz & 16 & $10.29(2.38)$ & 17 & $11.14(2.14)$ & $t(30)=-1.07, p=0.29, d=-0.37$ \\
\hline
\end{tabular}


Supplementary Table 2: Neural entrainment assessed through previous methods. Mean and standard deviation $(M(S D))$ of signal to noise ratio $(S N R)$, phase locking value (PLV), inter-hemispheric phase synchronization and coherence.

\begin{tabular}{|c|c|c|c|c|}
\hline & \multicolumn{2}{|c|}{ Control participants } & \multicolumn{2}{|c|}{ Dyslexic participants } \\
\hline & Left & Right & Left & Right \\
\hline \multicolumn{5}{|l|}{ SNR [dB] } \\
\hline $2 \mathrm{~Hz}$ & $8.23(6.01)$ & $9.48(6.70)$ & $6.84(5.10)$ & $10.41(7.94)$ \\
\hline $5 \mathrm{~Hz}$ & $9.85(5.94)$ & $8.56(4.50)$ & $6.66(3.81)$ & $6.70(3.89)$ \\
\hline $30 \mathrm{~Hz}$ & $8.89(5.77)$ & $10.27(7.4)$ & $7.77(5.59)$ & $8.20(6.34)$ \\
\hline \multicolumn{5}{|l|}{ PLV [0-1] } \\
\hline $2 \mathrm{~Hz}$ & $0.19(0.07)$ & $0.22(0.11)$ & $0.21(0.07)$ & $0.25(0.07)$ \\
\hline $5 \mathrm{~Hz}$ & $0.17(0.06)$ & $0.18(0.06)$ & $0.16(0.05)$ & $0.19(0.07)$ \\
\hline $30 \mathrm{~Hz}$ & $0.08(0.03)$ & $0.09(0.04)$ & $0.08(0.03)$ & $0.09(0.04)$ \\
\hline \multicolumn{5}{|c|}{ IHPS [0 - 1] } \\
\hline $2 \mathrm{~Hz}$ & \multicolumn{2}{|c|}{$0.15(0.19)$} & \multicolumn{2}{|c|}{$0.11(0.13)$} \\
\hline $5 \mathrm{~Hz}$ & \multicolumn{2}{|c|}{$0.13(0.20)$} & \multicolumn{2}{|c|}{$0.10(0.16)$} \\
\hline $30 \mathrm{~Hz}$ & \multicolumn{2}{|c|}{$0.17(0.20)$} & \multicolumn{2}{|c|}{$0.13(0.15)$} \\
\hline \multicolumn{5}{|c|}{ Coherence $[0-1]$} \\
\hline \multicolumn{5}{|c|}{ Forward speech } \\
\hline Delta & $0.04(0.01)$ & $0.04(0.01)$ & $0.04(0.01)$ & $0.04(0.01)$ \\
\hline Theta & $0.03(0.01)$ & $0.04(0.01)$ & $0.03(0.01)$ & $0.03(0.01)$ \\
\hline \multicolumn{5}{|c|}{ Backward speech } \\
\hline Delta & $0.04(0.01)$ & $0.04(0.01)$ & $0.04(0.01)$ & $0.04(0.01)$ \\
\hline Theta & $0.03(0.01)$ & $0.03(0.01)$ & $0.03(0.01)$ & $0.03(0.01)$ \\
\hline
\end{tabular}

Supplementary Table 3: Neural entrainment to nonspeech stimuli assessed through cross-correlation analysis. Mean and standard deviation $(M(S D))$ of the maximum phase correlation $\left(\rho_{\max }\right)$ and the corresponding lag $(\tau)$ values.

\begin{tabular}{|c|c|c|c|c|}
\hline & \multicolumn{2}{|c|}{ Control participants } & \multicolumn{2}{|c|}{ Dyslexic participants } \\
\hline & Left & Right & Left & Right \\
\hline \multicolumn{5}{|c|}{ Phase $\rho_{\max }[0-1]$} \\
\hline $2 \mathrm{~Hz}$ & $0.26(0.01)$ & $0.27(0.02)$ & $0.028(0.02)$ & $0.027(0.02)$ \\
\hline $5 \mathrm{~Hz}$ & $0.39(0.01)$ & $0.40(0.02)$ & $0.40(0.02)$ & $0.40(0.02)$ \\
\hline $30 \mathrm{~Hz}$ & $0.71(0.01)$ & $0.71(0.01)$ & $0.71(0.01)$ & $0.071(0.01)$ \\
\hline \multicolumn{5}{|c|}{ Phase $\tau[\mathrm{ms}]$} \\
\hline $2 \mathrm{~Hz}$ & $73.02(3.04)$ & 72.77 (3.64) & $74.51(2.81)$ & $73.69(2.89)$ \\
\hline $5 \mathrm{~Hz}$ & 73.83 (1.79) & 73.44 (2.52) & $73.23(2.05)$ & $73.30(2.91)$ \\
\hline $30 \mathrm{~Hz}$ & $75.03(2.31)$ & $74.78(2.55)$ & $75.41(2.07)$ & $74.83(2.29)$ \\
\hline
\end{tabular}


Supplementary Table 4: Neural entrainment to nonspeech stimuli assessed through cross-correlation analysis. Mean and standard deviation $(\mathrm{M}(\mathrm{SD}))$ of the maximum amplitude correlation $\left(\rho_{\max }\right)$ and the corresponding lag $(\tau)$ values.

\begin{tabular}{|c|c|c|c|c|}
\hline & \multicolumn{2}{|c|}{ Control participants } & \multicolumn{2}{c|}{ Dyslexic participants } \\
\hline & Left & Right & Left & Right \\
\hline \multicolumn{5}{|c|}{ Amplitude $\rho_{\max }[\mathbf{0}-\mathbf{1}]$} \\
\hline $2 \mathrm{~Hz}$ & $0.07(0.01)$ & $0.06(0.01)$ & $0.07(0.01)$ & $0.06(0.01)$ \\
\hline $5 \mathrm{~Hz}$ & $0.05(0.01)$ & $0.05(0.01)$ & $0.05(0.01)$ & $0.05(0.01)$ \\
\hline $30 \mathrm{~Hz}$ & $0.04(0.01)$ & $0.04(0.01)$ & $0.04(0.01)$ & $0.04(0.01)$ \\
\hline \multicolumn{5}{|c|}{ Amplitude $\mathbf{\tau}[\mathrm{ms}]$} \\
\hline $2 \mathrm{~Hz}$ & $73.85(1.81)$ & $73.96(1.86)$ & $73.82(2.18)$ & $74.35(2.25)$ \\
\hline $5 \mathrm{~Hz}$ & $74.65(1.71)$ & $75.36(2.21)$ & $74.85(1.34)$ & $74.59(1.38)$ \\
\hline $30 \mathrm{~Hz}$ & $80.14(1.56)$ & $79.84(2.55)$ & $81.15(1.40)$ & $80.97(1.20)$ \\
\hline
\end{tabular}

Supplementary Table 5: Neural entrainment to speech stimuli assessed through cross-correlation analysis. Mean and standard deviation $(M(S D))$ of the maximum phase correlation $\left(\rho_{\max }\right)$ and the corresponding lag $(\tau)$ values.

\begin{tabular}{|c|c|c|c|c|}
\hline & \multicolumn{2}{|c|}{ Control participants } & \multicolumn{2}{|c|}{ Dyslexic participants } \\
\hline & Left & Right & Left & Right \\
\hline \multicolumn{5}{|c|}{ Phase $\rho_{\max }[0-1]$} \\
\hline \multicolumn{5}{|c|}{ Forward speech } \\
\hline Delta & $0.204(0.004)$ & $0.198(0.004)$ & $0.203(0.004)$ & $0.198(0.003)$ \\
\hline Theta & $0.148(0.003)$ & $0.148(0.002)$ & $0.148(0.002)$ & $0.148(0.003)$ \\
\hline \multicolumn{5}{|c|}{ Backward speech } \\
\hline Delta & $0.201(0.004)$ & $0.201(0.003)$ & $0.201(0.003)$ & $0.202(0.004)$ \\
\hline Theta & $0.146(0.002)$ & $0.147(0.002)$ & $0.149(0.003)$ & $0.148(0.004)$ \\
\hline \multicolumn{5}{|c|}{ 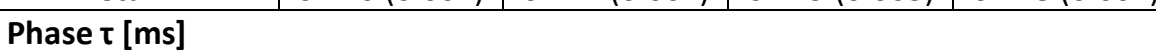 } \\
\hline \multicolumn{5}{|c|}{ Forward speech } \\
\hline Delta & $75.51(1.81)$ & $75.17(1.71)$ & $74.74(1.94)$ & 74.99 (1.62) \\
\hline Theta & $74.84(1.59)$ & $75.17(1.55)$ & $75.28(1.21)$ & $75.26(0.92)$ \\
\hline \multicolumn{5}{|c|}{ Backward speech } \\
\hline Delta & $75.86(2.24)$ & $75.57(2.65)$ & $75.10(1.19)$ & $75.91(2.03)$ \\
\hline Theta & 75.51 (1.19) & $75.48(1.17)$ & $75.04(1.64)$ & $75.38(1.01)$ \\
\hline
\end{tabular}

Supplementary Table 6: Neural entrainment to speech stimuli assessed through cross-correlation analysis. Mean and standard deviation $(\mathrm{M}(\mathrm{SD}))$ of the maximum amplitude correlation $\left(\rho_{\max }\right)$ and the corresponding lag $(\tau)$ values.

\begin{tabular}{|l|c|c|c|c|}
\hline & \multicolumn{2}{|c|}{ Control participants } & \multicolumn{2}{|c|}{ Dyslexic participants } \\
\hline & Left & Right & Left & Right \\
\hline \multicolumn{2}{|l}{} \\
\hline \multicolumn{2}{|l}{ Amplitude $\rho_{\max }[0-1]$}
\end{tabular}




\begin{tabular}{|c|c|c|c|c|}
\hline \multicolumn{5}{|c|}{ Forward speech } \\
\hline Delta & $0.211(0.005)$ & $0.211(0.005)$ & $0.211(0.004)$ & $0.210(0.004)$ \\
\hline Theta & $0.212(0.004)$ & $0.211(0.004)$ & $0.210(0.003)$ & $0.211(0.004)$ \\
\hline Alpha & $0.209(0.006)$ & $0.208(0.005)$ & $0.209(0.003)$ & $0.210(0.005)$ \\
\hline Beta & $0.207(0.003)$ & $0.207(0.003)$ & $0.208(0.002)$ & $0.207(0.003)$ \\
\hline Gamma & $0.206(0.003)$ & $0.206(0.002)$ & $0.206(0.002)$ & $0.207(0.002)$ \\
\hline \multicolumn{5}{|c|}{ Backward speech } \\
\hline Delta & $0.209(0.005)$ & $0.209(0.004)$ & $0.210(0.003)$ & $0.210(0.003)$ \\
\hline Theta & $0.210(0.005)$ & $0.211(0.004)$ & $0.210(0.004)$ & $0.209(0.004)$ \\
\hline Alpha & $0.210(0.005)$ & $0.211(0.004)$ & 0.209 (0.005) & $0.209(0.005)$ \\
\hline Beta & $0.208(0.002)$ & $0.208(0.003)$ & $0.207(0.002)$ & $0.206(0.003)$ \\
\hline Gamma & $0.206(0.003)$ & $0.206(0.004)$ & $0.205(0.002)$ & $0.206(0.003)$ \\
\hline \multicolumn{5}{|c|}{ Amplitude $\tau$ [ms] } \\
\hline \multicolumn{5}{|c|}{ Forward speech } \\
\hline Delta & 75.19 (2.25) & $75.48(2.02)$ & 73.46 (1.67) & $74.31(1.26)$ \\
\hline Theta & 74.25 (1.39) & $74.68(1.52)$ & 74.51 (1.63) & $74.25(2.02)$ \\
\hline Alpha & $75.53(2.06)$ & $75.27(1.95)$ & 75.19 (1.63) & $75.15(1.53)$ \\
\hline Beta & 75.46 (1.37) & $75.52(1.15)$ & 74.75 (1.48) & 74.89 (1.59) \\
\hline Gamma & 74.54 (1.98) & $75.11(1.50)$ & $74.93(1.37)$ & 74.91 (1.19) \\
\hline \multicolumn{5}{|c|}{ Backward speech } \\
\hline Delta & $75.01(1.85)$ & 74.92 (1.17) & 75.21 (1.68) & $74.61(1.66)$ \\
\hline Theta & $74.83(1.35)$ & $75.09(1.96)$ & $74.98(1.74)$ & $75.19(2.04)$ \\
\hline Alpha & $74.98(2.19)$ & $75.12(1.31)$ & $75.25(2.21)$ & $75.03(0.89)$ \\
\hline Beta & 74.87 (1.08) & $75.54(1.78)$ & $74.65(0.89)$ & $75.03(0.84)$ \\
\hline Gamma & $75.26(1.86)$ & $75.87(1.37)$ & 74.77 (1.78) & $74.71(1.13)$ \\
\hline
\end{tabular}

